\title{
ON NEW EXAMPLES OF BALLISTIC RANDOM WALKS IN RANDOM ENVIRONMENT
}

\author{
BY ALAIN-SOL SZNITMAN
}

\section{ETH-Zentrum}

\begin{abstract}
In this article we show that random walks in random environment on $\mathbb{Z}^{d}$, $d \geq 3$, with transition probabilities which are $\varepsilon$-perturbations of the simple random walk and such that the expectation of the local drift has size bigger than $\varepsilon^{\rho}$, with $\rho<\frac{5}{2}$, when $d=3, \rho<3$, when $d \geq 4$, fulfill the condition $\left(\mathrm{T}^{\prime}\right)$ introduced by Sznitman [Prob. Theory Related Fields (2002) 122 509-544], when $\varepsilon$ is small. As a result these walks satisfy a law of large numbers with nondegenerate limiting velocity, a central limit theorem and several large deviation controls. In particular, this provides examples of ballistic random walks in random environment which do not satisfy Kalikow's condition in the terminology of Sznitman and Zerner [Ann. Probab. (1999) 27 1851-1869]. An important tool in this work is the effective criterion of Sznitman.
\end{abstract}

0. Introduction. Random walks in random environment on $\mathbb{Z}^{d}$ constitute one of the basic models of random motions in a random medium; see Hughes [6], Molchanov [12] and Zeitouni [23]. Yet in dimension bigger than one, the present understanding of their asymptotic behavior is rather primitive. As opposed to other models of random motions in random media (see, e.g., [2, 14, 9, 13]), until about three years ago few references dealt with their study (cf. Kalikow [7], Lawler [10] and Bricmont and Kupiainen [5]). Recently however, there has been some progress in the understanding of random walks in random environment with ballistic behavior (cf. [3] for a review). In [20] the so-called condition (T) and in [21] the slightly weaker condition $\left(\mathrm{T}^{\prime}\right)$ were introduced [we recall what these conditions are in (0.3)]. As soon as the dimension $d$ is bigger than 1, these conditions have a number of interesting consequences such as a ballistic law of large numbers, a central limit theorem and several large deviation controls, which in particular hold for certain critical regimes related to slowdowns of the walk. In fact, there is a strong suspicion that $(T)$ and $\left(\mathrm{T}^{\prime}\right)$ are equivalent. They may even turn out to characterize ballistic behaviors of random walks in random environment when $d \geq 2$.

In [21] condition ( $\left.\mathrm{T}^{\prime}\right)$ was shown to be equivalent to an effective criterion, which can be checked by direct inspection of the environment in a finite box of large size. The main purpose of the present article is to present an application of this effective criterion which provides new examples of walks with ballistic behavior. These

Received August 2001; revised February 2002.

AMS 2000 subject classifications. 60K37, 82D30.

Key words and phrases. Random walk in random environment, ballistic behavior, small perturbations of simple random walk. 
walks come as small perturbations of the simple random walk on $\mathbb{Z}^{d}, d \geq 3$, and offer instances of ballistic behavior beyond current knowledge.

Before discussing these examples any further we recall the model. The environment where the walk evolves is described by a collection of i.i.d. (2d)-dimensional vectors, which prescribe the transition probability of the walk at each site of $\mathbb{Z}^{d}$ ( $d \geq 1$, for the time being). We implicitly assume ellipticity of the motion, and for some $\kappa \in\left(0, \frac{1}{2 d}\right]$,

The common law $\mu$ of the vectors is supported on $\mathcal{P}_{\kappa}$, the set of $(2 d)$-vectors $(p(e))_{|e|=1, e \in \mathbb{Z}^{d}}$, with $p(e) \in[\kappa, 1]$, for each $e$ and $\sum_{|e|=1} p(e)=1$.

The random environment is then an element $\omega=(\omega(x, \cdot))_{x \in \mathbb{Z}^{d}}$ of $\Omega=\mathcal{P}_{\kappa}^{\mathbb{Z}^{d}}$, which is endowed with the product $\sigma$-algebra and the product measure $\mathbb{P}=\mu^{\otimes \mathbb{Z}^{d}}$. The random walk in the random environment $\omega$ is the canonical Markov chain $\left(X_{n}\right)_{n \geq 0}$ on $\left(\mathbb{Z}^{d}\right)^{\mathbb{N}}$, with state space $\mathbb{Z}^{d}$, "quenched" law $P_{x, \omega}$ starting from $x \in \mathbb{Z}^{d}$, and transition probability $\omega(y, e)$ of being in $y+e$ at the next step, when in $y$. One also defines for $x \in \mathbb{Z}^{d}$, the "annealed" laws $P_{x}$ on $\Omega \times\left(\mathbb{Z}^{d}\right)^{\mathbb{N}}$, as the semidirect product

$$
P_{x}=\mathbb{P} \times P_{x, \omega} .
$$

We now turn to conditions (T) and $\left(\mathrm{T}^{\prime}\right)$ of [20] and [21]. We only discuss here one of the equivalent characterizations of $(\mathrm{T})$ and $\left(\mathrm{T}^{\prime}\right)$ in terms of decays of certain exit probabilities of the walk from thick slabs. We refer to [21] for other equivalent formulations. If $\ell \in S^{d-1}$ is a unit vector of $\mathbb{R}^{d}$, we say that $\left(\mathrm{T}^{\prime}\right)$ holds relative to $\ell$ if for every $\gamma \in(0,1)$, for all $\ell^{\prime}$ in some neighborhood of $\ell$,

$$
\limsup _{L \rightarrow \infty} L^{-\gamma} \log P_{0}\left[\widetilde{T}_{-b L}^{\ell^{\prime}}<T_{L}^{\ell^{\prime}}\right]<0 \quad \text { for any } b>0
$$

where for $w \in S^{d-1}, u \in \mathbb{R}$,

$$
T_{u}^{w}=\inf \left\{n \geq 0, X_{n} \cdot w \geq u\right\}, \quad \widetilde{T}_{u}^{w}=\inf \left\{n \geq 0, X_{n} \cdot w \leq u\right\} .
$$

In a similar fashion, condition (T) is said to hold relative to $\ell$ when for all $\ell^{\prime}$ in some neighborhood of $\ell,(0.3)$ holds with $\gamma=1$. Clearly, condition (T) relative to $\ell$ implies condition $\left(\mathrm{T}^{\prime}\right)$ relative to $\ell$. There is a strong suspicion that both conditions are equivalent. As soon as $d \geq 2,\left(\mathrm{~T}^{\prime}\right)$ relative to $\ell$ implies a ballistic law of large numbers,

$$
P_{0} \text {-a.s., } \frac{X_{n}}{n} \rightarrow v, v \text { deterministic with } v \cdot \ell>0,
$$

a central limit theorem,

(0.6) on the Skorohod space $D\left(\mathbb{R}_{+}, \mathbb{R}^{d}\right)$ converge weakly to a Brownian motion with a nondegenerate covariance matrix, 
and several large deviation controls on $\frac{X_{n}}{n}$ (slightly weaker than the corresponding results under condition (T), cf. [20, 21]). Incidentally these controls are more delicate than (0.5) and (0.6), and show that for a broad class of walks large deviations "on the segment $[0, v]$ " are critical. Such large deviations are closely linked to slowdowns of the walk.

In this article we consider certain small perturbations of the simple random walk on $\mathbb{Z}^{d}$, when $d \geq 3$. More precisely, for $\varepsilon \in(0,1)$ we define

$$
\begin{aligned}
& \oiint_{\varepsilon} \text { the subset of } \mathcal{P}_{\kappa=1 / 4 d} \text { of }(2 d) \text {-vectors }(p(e))_{|e|=1} \\
& \text { with }\left|p(e)-\frac{1}{2 d}\right| \leq \frac{\varepsilon}{4 d}, \text { for each } e,
\end{aligned}
$$

and denote by $\Omega_{\varepsilon}$ the subset $\rho_{\varepsilon}^{\otimes \mathbb{Z}^{d}}$ of $\Omega$ (with $\kappa=\frac{1}{4 d}$ ). The local drift at site $x$ in the environment $\omega$ is defined as

$$
d(x, \omega)=\sum_{|e|=1} \omega(x, e) e .
$$

The main result of the present article is the following.

THEOREM $(d \geq 3)$. For $\eta \in(0,1)$, there exists $\varepsilon_{0}(d, \eta) \in(0,1)$ such that for $0<\varepsilon<\varepsilon_{0}$, whenever the single site distribution $\mu$ is supported on $\S_{\varepsilon}$ and

$$
\lambda \stackrel{\text { def }}{=} \mathbb{E}\left[d(0, \omega) \cdot e_{1}\right]> \begin{cases}\varepsilon^{5 / 2-\eta}, & \text { when } d=3, \\ \varepsilon^{3-\eta}, & \text { when } d \geq 4,\end{cases}
$$

condition $\left(\mathrm{T}^{\prime}\right)$ holds with respect to $e_{1}$ the first coordinate vector of the canonical basis of $\mathbb{R}^{d}$. The expectation in (0.9) is taken relative to $\mathbb{P}\left(=\mu^{\otimes \mathbb{Z}^{d}}\right)$.

The above theorem reads off the ballistic nature of the walk from the expectation of $d(0, \omega)$ with respect to the straightforward static distribution $\mathbb{P}$, instead of a dynamic distribution stemming from the method of the "environment viewed from the particle" (cf. $[9,12,13,15])$. It provides new instances of ballistic behavior. In particular, it gives examples of walks for which $\left(\mathrm{T}^{\prime}\right)$ holds but the so-called Kalikow's condition breaks down; cf. [7, 22] and (5.3). For instance when $d \geq 3$ and $\tilde{\mu}$ supported on $\delta_{\varepsilon / 2}$ is isotropic, that is, invariant under rotations of $\mathbb{R}^{d}$ preserving $\mathbb{Z}^{d}$, and for some $\rho \in(0,1)$,

$$
\operatorname{var}_{\tilde{\mu}}\left(p\left(e_{1}\right)\right) \geq \rho \varepsilon^{2}, \quad \operatorname{cov}_{\widetilde{\mu}}\left(p\left(e_{1}\right), p\left(-e_{1}\right)\right) \leq(1-\rho) \operatorname{var}_{\tilde{\mu}}\left(p\left(e_{1}\right)\right),
$$

it follows from Theorem 5.1 that the image $\mu$ of $\tilde{\mu}$ under the map

$$
(p(e))_{|e|=1} \rightarrow\left(p(e)+\frac{\lambda}{2} e \cdot e_{1}\right)_{|e|=1} \quad \text { with } \lambda \in\left(0, c(d, \rho) \varepsilon^{2}\right)
$$

does not satisfy Kalikow's condition with respect to any direction for small $\varepsilon$ (depending on $d, \rho)$. However, when $\lambda$ is not too small [cf. (0.9)], condition $\left(\mathrm{T}^{\prime}\right)$ 
relative to $e_{1}$ is fulfilled. The above distribution $\mu$ can be viewed as a slight anisotropic perturbation of the isotropic perturbation $\tilde{\mu}$ of the simple random walk (such isotropic perturbations are considered in [5]). This is only one possible example. The main result applies to more general situations.

One can naturally wonder whether the conclusion of the theorem holds when we replace (0.9) with the weaker assumption $\lambda>0$. This is not the case and we refer to [4], where examples of slight perturbations of the simple random walk with diffusive behavior and yet nonvanishing $\lambda$ are constructed.

The proof of our main result relies on the effective criterion of [21]. Indeed, in the case of direction $e_{1}$, it is proved in Theorem 2.4 of [21] that $\left(\mathrm{T}^{\prime}\right)$ relative to $e_{1}$ is equivalent to

$$
\inf _{B_{0}, a}\left\{c(d)\left(\log \frac{1}{\kappa}\right)^{3(d-1)} \widetilde{L}_{0}^{(d-1)} L_{0}^{3(d-1)+1} \mathbb{E}\left[\rho_{B_{0}}^{a}\right]\right\}<1,
$$

where $B_{0}$ runs over all boxes $\left(-L_{0}+2, L_{0}+2\right) \times\left(-\widetilde{L}_{0}, \widetilde{L}_{0}\right)^{d-1}$, with $L_{0} \geq c^{\prime}(d)$, $3 \sqrt{d} \leq \widetilde{L}_{0}<L_{0}^{3}, a \in(0,1]$, and $c(d)$ and $c^{\prime}(d)$ are some explicit positive dimension dependent constants,

$$
\rho_{B_{0}}=\frac{P_{0, \omega}\left[X_{T_{B_{0}}} \notin \partial_{+} B_{0}\right]}{P_{0, \omega}\left[X_{T_{B_{0}}} \in \partial_{+} B_{0}\right]},
$$

provided $T_{B_{0}}$ stands for the exit time from $B_{0}$ and

$$
\partial_{+} B_{0}=\left\{z \in \mathbb{Z}^{d} \backslash B_{0}, \exists z^{\prime} \in B_{0}\right. \text { such that }
$$

$$
\left.\left|z-z^{\prime}\right|=1, z \cdot e_{1} \geq L_{0}+2,\left|z \cdot e_{i}\right|<\tilde{L}_{0} \text { for } i \geq 2\right\} .
$$

We recall that in the present setting $\kappa=\frac{1}{4 d}$. Intuitively $(0.12)$ has some flavor of the condition $\mathbb{E}[\rho]<1,(\rho=\omega(0,-1) / \omega(0,1))$, which in the one-dimensional context characterizes ballistic walks with positive velocity (cf. Solomon [17]). Note, however, that $(0.12)$ involves moments of $\rho_{B_{0}}$ of arbitrarily small order. This can be viewed as a reflection of the weakening effect of traps in higher dimension.

To prove the main result, we check $(0.12)$ with $L_{0}$ of order $\varepsilon^{-4}$ and $\widetilde{L}_{0}$ of order $L_{0}^{3}$. We derive in Corollary 1.3 a general upper bound on $\mathbb{E}\left[\rho_{B_{0}}^{a}\right]$. In the present context this bound enables us to control the potentially small denominator in $\rho_{B_{0}}^{a}$, which is linked to the occurrence of atypical exit distributions of the walk from $B_{0}$. This feature is closely related to the presence of traps in the medium (cf. [20]). The general bound derived in Section 1 reduces the task of checking $(0.12)$ to the control of the random variable

$$
D=E_{0, \omega}\left[\sum_{k=0}^{T_{U}-1} d\left(X_{k}, \omega\right) \cdot e_{1}\right]
$$

where $d(x, \omega)$ is defined in $(0.8)$ and $T_{U}$ denotes the exit time from the strip

$$
U=\left\{z \in \mathbb{Z}^{d},\left|z \cdot e_{1}\right|<L\right\}
$$


( $L$ is an integer comparable to $\varepsilon^{-1}$ ). To check $(0.12)$ it then suffices to prove that $D$ has $\mathbb{P}$-expectation bigger than const $\lambda L^{2}$ but fluctuations of a smaller size. The advantage is that one now works in $U$ where the walk in the environment $\omega \in \Omega_{\varepsilon}$ cannot deviate too much from the simple random walk. In the terminology of Bricmont and Kupiainen [5], we only need to deal with a "small field problem."

Of course $D$ can be re-expressed in terms of the Green function of the walk killed outside $U$,

$$
g_{U}(x, y, \omega)=E_{x, \omega}\left[\sum_{0}^{T_{U}-1} \mathbb{1}\left\{X_{k}=y\right\}\right],
$$

which can be compared to the corresponding Green function $g_{0, U}(\cdot, \cdot)$ of the simple random walk killed outside $U$. In Section 3 we derive in Propositions 3.1 and 3.2 the required estimates on the expectation of $D$ and on the size of its fluctuations. The difficulty is that $d(x, \omega) \cdot e_{1}$ typically has size of order $\varepsilon$, which is potentially much larger than its expectation $\lambda$ [cf. (0.9)]. One needs to show that cancellations take place. We then control the fluctuations of $D$ by the martingale method, and show in Proposition 3.2 that for $\gamma \in(1,2]$ and small $\varepsilon$ ( $L$ depends on $d, \gamma, \varepsilon$, and $\gamma$ close to 1 is the more interesting choice),

$$
\begin{aligned}
& \mathbb{P}[|D-\mathbb{E}[D]|>u] \leq 2 \exp \left\{-\frac{u^{2}}{\sigma^{2}}\right\} \quad \text { for } u>0, \\
& \text { with } \sigma^{2} \leq c(d) \varepsilon^{2} \sum_{y \in U} g_{0, U}(0, y)^{2 / \gamma} \leq c(d, \gamma) \varepsilon^{v(d, \gamma)} \quad \text { where }
\end{aligned}
$$

$$
v(d, \gamma)= \begin{cases}1-\frac{2(\gamma-1)}{\gamma}, & \text { when } d=3, \\ 2-\frac{4(\gamma-1)}{\gamma}, & \text { when } d=4, \\ 2, & \text { when } d \geq 5 \text { and } \gamma<\frac{6}{5} .\end{cases}
$$

Let us finally explain the structure of this article.

In Section 1, we introduce some further notations and derive a general bound of independent interest that enables controlling $\mathbb{E}\left[\rho_{B_{0}}^{a}\right]$. In Section 2 , we collect deterministic estimates on $g_{U}(\cdot, \cdot, \omega), \omega \in \Omega_{\varepsilon}$, and on $g_{0, U}(\cdot, \cdot)$. Section 3 is devoted to the derivation of a lower bound on $\mathbb{E}[D]$ and an upper bound on the fluctuations of $D$. Section 4 combines the estimates of the previous sections to prove the main result. Section 5 recalls the definition of Kalikow's condition and provides examples of walks for which $\left(\mathrm{T}^{\prime}\right)$ holds but Kalikow's condition fails. Finally, the Appendix contains a proof of Lemma 1.2, which is used in Section 1, and of certain Green function estimates for the simple random walk killed outside the slab $U$. 
1. A bound on fractional moments of $\rho_{B_{0}}(d \geq 2)$. In this section we first introduce some further notations. The main object, however, is to derive a general estimate on fractional moments of $\rho_{B_{0}}$ (cf. Corollary 1.3). This estimate will later be applied in Section 4 when proving our main result [cf. (0.9) or Theorem 4.1]. For the main estimate of this section we slice $B_{0}$ into layers along the first axis and we control $\mathbb{E}\left[\rho_{B_{0}}^{a}\right]$ in terms of quantities in a single layer. This has of course the same flavor as the main renormalization step derived in Proposition 2.1 of [21]. However, the estimates are conducted in a different fashion because we are looking here for a seed estimate of the renormalization scheme in [21] [i.e., checking (0.12)], whereas in [21] the objective was to set up a renormalization scheme.

We begin with additional notations. We denote by $|\cdot|$ the Euclidean distance on $\mathbb{R}^{d}$, and write $B(w, r)$ for the open $|\cdot|$-ball of radius $r$ centered at $w \in \mathbb{R}^{d}$. For $U$, a subset of $\mathbb{Z}^{d},|U|$ stands for the cardinality of $U$, and $\partial U$ for the boundary of $U$,

$$
\partial U=\left\{x \in \mathbb{Z}^{d} \backslash U, \exists y \in U,|x-y|=1\right\} .
$$

We denote by $\left(t_{x}\right)_{x \in \mathbb{Z}^{d}}$ the spatial shift on $\Omega=\mathcal{P}_{\kappa}^{\mathbb{Z}^{d}}$ and by $\left(\theta_{n}\right)_{n \geq 0}$ the canonical shift on $\left(\mathbb{Z}^{d}\right)^{\mathbb{N}}$. We write $\left(\mathcal{F}_{n}\right)_{n \geq 0}$ for the canonical filtration on $\left(\mathbb{Z}^{d}\right)^{\mathbb{N}}$ attached to the canonical process $\left(X_{n}\right)_{n \geq 0}$. For $U \subseteq \mathbb{Z}^{d}, H_{U}$ and $T_{U}$ stand for the respective entrance and exit time of $X_{n}$ in (resp. from) $U$

$$
H_{U}=\inf \left\{n \geq 0, X_{n} \in U\right\}, \quad T_{U}=\inf \left\{n \geq 0, X_{n} \notin U\right\} .
$$

We consider $L>1, N>1$, even integers, as well as the box

$$
B=(-N L, N L) \times\left(-\frac{1}{4}(N L)^{3}, \frac{1}{4}(N L)^{3}\right)^{d-1} .
$$

It may be helpful to mention that the box $B_{0}$ mentioned above is simply $B_{0}=B+2 e_{1}$, so that in the notation below (0.12), $L_{0}=N L$ and $\widetilde{L}_{0}=\frac{1}{4}(N L)^{3}$. We define

$$
\begin{aligned}
p_{B}(\omega) & =1-q_{B}(\omega) \\
& =P_{0, \omega}\left[X_{T_{B}} \cdot e_{1} \geq N L,\left|X_{T_{B}} \cdot e_{i}\right|<\frac{1}{4}(N L)^{3} \text { for all } i \geq 2\right], \\
\rho_{B} & =\frac{q_{B}}{p_{B}} .
\end{aligned}
$$

Further, for $x \in \mathbb{Z}^{d}, \omega \in \Omega, i \in \mathbb{Z}$, we introduce

$$
\widehat{p}(x, \omega)=1-\widehat{q}(x, \omega)=P_{x, \omega}\left[T_{x \cdot e_{1}+L}<\widetilde{T}_{x \cdot e_{1}-L}\right],
$$

where for $u$ in $\mathbb{R}, T_{u}, \widetilde{T}_{u}$ are defined as in (0.4) with the choice $w=e_{1}$, and

$$
\widehat{\rho}(i, \omega)=\sup \left\{\frac{\widehat{q}(x, \omega)}{\widehat{p}(x, \omega)}, x \in \mathscr{H}_{i}, \sup _{2 \leq j \leq d}\left|x \cdot e_{j}\right|<\frac{1}{4}(N L)^{3}\right\}
$$


with

$$
\mathscr{H}_{i}=\left\{x \in \mathbb{Z}^{d}, x \cdot e_{1}=i L\right\} .
$$

The quantities $\widehat{\rho}(i, \omega)$ enable us to mimic the construction of certain martingales which appear in the one-dimensional context [cf. (A.3) and (A.9) in the Appendix].

Our main purpose is to derive an upper bound on $\mathbb{E}\left[\rho_{B}^{a}\right], 0<a \leq 1$, which involves $\mathbb{E}\left[\hat{\rho}(0, \omega)^{2 a}\right]$ and some other quantities which we now introduce. We consider positive integers $h, H, M$ with

$$
2 h \leq H<\frac{1}{32}(N L)^{3} \text { and } M=\left[\frac{(N L)^{3}}{32 H}\right] .
$$

We introduce the stopping time

$$
S=\inf \left\{n \geq 0,\left|\left(X_{n}-X_{0}\right) \cdot e_{1}\right| \geq L \text { or } \sup _{j \geq 2}\left|\left(X_{n}-X_{0}\right) \cdot e_{j}\right| \geq h\right\},
$$

which will be used to compare displacements in the $e_{1}$ direction to displacements in the orthogonal directions. We also need the driftlike quantity

$$
\Delta(x, \omega)=E_{x, \omega}\left[X_{S}\right]-x, \quad x \in \mathbb{Z}^{d}, \omega \in \Omega .
$$

We can now define for $\gamma_{1}, \gamma_{2} \in(0,1]$,

$$
\begin{array}{r}
p=\inf _{j \geq 2} \mathbb{P}\left[\text { for all } z \in \widetilde{B}^{j}, \Delta(z, \omega) \cdot e_{1} \geq \gamma_{1} L,\right. \\
\left.\qquad\left|\Delta(z, \omega) \cdot e_{i}\right| \leq \gamma_{2} h \text { for all } i \geq 2\right],
\end{array}
$$

where for $2 \leq j \leq d$,

$$
\widetilde{B}^{j}=\left\{y \in B,\left|y \cdot e_{j}\right|<H\right\} .
$$

Incidentally, let us mention that for the applications in the present article we will only use the value $\gamma_{2}=1$. The principal bound comes in the following.

THEOREM $1.1[d \geq 2$, under (0.1)]. Assume $0<a \leq 1$ and

$$
\delta^{-1} \stackrel{\text { def }}{=} \exp \left\{-\frac{\gamma_{1} N}{32}\right\}+\frac{10 N}{\gamma_{1}} \exp \left\{-\frac{\gamma_{1} N}{32}\left(\frac{H}{2 h N}-\frac{4 \gamma_{2}}{\gamma_{1}}\right)_{+}^{2}\right\}<1
$$

then

$$
\begin{aligned}
\mathbb{E}\left[\rho_{B}^{a}\right] \leq & \frac{2 \mathbb{E}\left[\widehat{\rho}(0)^{2 a}\right]^{N / 2}}{\left(1-\mathbb{E}\left[\hat{\rho}(0)^{2 a}\right]^{1 / 2}\right)_{+}} \\
& +2 d \kappa^{-a N L} \exp \left\{-\frac{M}{2}\left(p-\frac{7 N L}{M} \frac{\log \kappa^{-1}}{\log \delta}\right)_{+}^{2}\right\}
\end{aligned}
$$


PROOF. We introduce

$$
\widetilde{T}=\inf \left\{n \geq 0, \sup _{i \geq 2}\left|X_{n} \cdot e_{i}\right| \geq \frac{1}{4}(N L)^{3}\right\}
$$

and

$$
g=\left\{\omega \in \Omega, P_{0, \omega}\left[\tilde{T} \leq \tilde{T}_{-N L} \wedge T_{N L}\right] \leq \kappa^{6 N L}\right\} .
$$

The first step comes with the following lemma.

LEMMA 1.2.

$$
\mathbb{E}\left[\rho_{B}^{a}\right] \leq \frac{2 \mathbb{E}\left[\widehat{\rho}(0)^{2 a}\right]^{N / 2}}{\left(1-\mathbb{E}\left[\widehat{\rho}(0)^{2 a}\right]^{1 / 2}\right)_{+}}+\kappa^{-a N L} \mathbb{P}\left[\mathcal{g}^{c}\right]
$$

[(1.14) is not needed for Lemma 1.2.]

The proof of Lemma 1.2 is essentially a variation on the derivation of (2.39) in [21]. For the reader's convenience we provide the proof of Lemma 1.2 in the Appendix.

Hence to prove $(1.15)$, our main task is to bound $\mathbb{P}\left[\mathcal{g}^{c}\right]$, and here we adopt a different strategy than in the proof of Proposition 2.1 of [21]. We denote by $S_{k}$, $k \geq 0$, the iterates of the stopping time $S$ introduced in (1.10), namely,

$$
S_{0}=0 \quad \text { and } \quad S_{k+1}=S \circ \theta_{S_{k}}+S_{k} \quad \text { for } k \geq 0 .
$$

Observe that for any $x$ and $\omega$, under $P_{x, \omega}$,

$$
M_{k}=X_{S_{k}}-X_{0}-\sum_{0}^{k-1} \Delta\left(X_{S_{m}}, \omega\right) \quad \text { is a }\left(\mathcal{F}_{S_{k}}\right) \text {-martingale. }
$$

Further, the increments of $M_{k} \cdot e_{i}$ are bounded by $2 L$ when $i=1$ and by $2 h$ when $i \geq 2$. Then for $\omega \in \Omega$, in the notations of (0.4),

$$
\begin{aligned}
P_{0, \omega}[ & \left.\widetilde{T} \leq \widetilde{T}_{-N L} \wedge T_{N L}\right] \\
& \leq \sum_{j=2}^{d}\left(P_{0, \omega}\left[T_{(1 / 8)(N L)^{3}}^{e_{j}}<T_{B}\right]+P_{0, \omega}\left[\widetilde{T}_{-(1 / 8)(N L)^{3}}^{e_{j}}<T_{B}\right]\right) .
\end{aligned}
$$

We now bound the term $P_{0, \omega}\left[T_{1 / 8(N L)^{3}}^{e_{2}}<T_{B}\right]$, all other terms can be handled in a similar fashion,

$$
\begin{aligned}
& P_{0, \omega}\left[T_{(1 / 8)(N L)^{3}}^{e_{2}}<T_{B}\right] \\
& \leq P_{0, \omega}\left[T_{4 M H}^{e_{2}}<T_{B}\right] \\
& \leq E_{0, \omega}\left[T_{4(M-1) H}^{e_{2}}<T_{B},\right.
\end{aligned}
$$

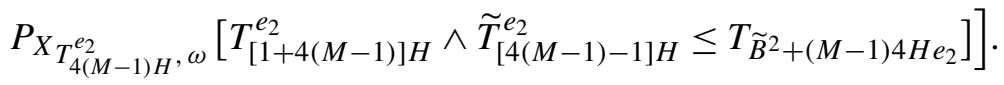


We now concentrate on the inner probability in the rightmost term of (1.22).

Note that for $x \in B$ with $\left|x \cdot e_{2}\right| \leq M 4 H$, and $t=\left[\frac{3 N}{\gamma_{1}}\right]+1$,

$$
\begin{aligned}
& P_{x, \omega}\left[T_{x \cdot e_{2}+H}^{e_{2}} \wedge \widetilde{T}_{x \cdot e_{2}-H}^{e_{2}} \leq T_{\widetilde{B}^{2}+x \cdot e_{2} e_{2}}\right] \\
& \quad \leq P_{x, \omega}\left[T_{x \cdot e_{2}+H}^{e_{2}} \wedge \widetilde{T}_{x \cdot e_{2}-H}^{e_{2}} \leq S_{t} \wedge T_{\widetilde{B}^{2}+x \cdot e_{2} e_{2}}\right]+P_{x, \omega}\left[S_{t}<T_{\widetilde{B}^{2}+x \cdot e_{2} e_{2}}\right] .
\end{aligned}
$$

We first bound the rightmost term. For $x$ as above,

$$
\begin{aligned}
P_{x, \omega} & {\left[S_{t}<T_{\widetilde{B}^{2}+x \cdot e_{2} e_{2}}\right] } \\
& \leq P_{x, \omega}\left[S_{t}<T_{\widetilde{B}^{2}+x \cdot e_{2} e_{2}}, M_{t} \cdot e_{1}+\sum_{0}^{t-1} \Delta\left(X_{S_{k}}, \omega\right) \cdot e_{1} \leq 2 N L\right] .
\end{aligned}
$$

Assume that $\omega$ is such that

(1.24) for all $z \in \widetilde{B}^{2}+x \cdot e_{2} e_{2}$,

$$
\Delta(z, \omega) \cdot e_{1} \geq \gamma_{1} L, \sup _{j \geq 2}\left|\Delta(z, \omega) \cdot e_{j}\right| \leq \gamma_{2} h,
$$

then the above quantity is smaller than

$$
P_{x, \omega}\left[M_{t} \cdot e_{1} \leq 2 N L-\gamma_{1} L t\right] \leq \exp \left\{-\frac{\left(2 N-\gamma_{1} t\right)_{-}^{2}}{8 t}\right\}
$$

in view of Azuma's inequality (cf. Alon, Spencer and Erdös [1], page 85), and the remark below (1.20). Since $\frac{3 N}{\gamma_{1}} \leq t \leq \frac{4 N}{\gamma_{1}}$, the above is smaller than $\exp \left\{-\frac{\gamma_{1} N}{32}\right\}$.

We then bound the first term in the right-hand side of (1.23), which is smaller than

$$
P_{x, \omega}\left[S_{I} \wedge S_{\widetilde{I}} \leq S_{t} \wedge T_{\widetilde{B}^{2}+x \cdot e_{2} e_{2}}\right]
$$

provided

$$
\begin{aligned}
& I=\inf \left\{k \geq 0, X_{S_{k}} \cdot e_{2}-x \cdot e_{2} \geq H-h\right\}, \\
& \tilde{I}=\inf \left\{k \geq 0, X_{S_{k}} \cdot e_{2}-x \cdot e_{2} \leq h-H\right\}
\end{aligned}
$$

(note that $P_{x, \omega}$-a.s., $I \geq 1, \widetilde{I} \geq 1$ and $S_{I} \wedge S_{\widetilde{I}} \leq T_{x \cdot e_{2}+H}^{e_{2}} \wedge \widetilde{T}_{x \cdot e_{2}-H}^{e_{2}}$ ). The above quantity is smaller than

$$
\begin{aligned}
& P_{x, \omega}\left[I \leq t, S_{I} \leq T_{\widetilde{B}^{2}+x \cdot e_{2} e_{2}}, M_{I} \cdot e_{2}+\sum_{0}^{I-1} \Delta\left(X_{S_{k}}, \omega\right) \cdot e_{2} \geq H-h\right] \\
& \quad+\text { same term with } \widetilde{I} \text { in place of } I \text { and " } \leq h-H \text { " in place of " } \geq H-h "
\end{aligned}
$$

and if (1.24) holds, noting that $t+1 \leq \frac{5 N}{\gamma_{1}}$, by Azuma's inequality,

$$
\leq 2(T+1) \exp \left\{-\frac{1}{8 t}\left(\frac{H}{h}-1-\gamma_{2} t\right)_{+}^{2}\right\} \stackrel{(1.9)}{\leq} \frac{10 N}{\gamma_{1}} \exp \left\{-\frac{\gamma_{1} N}{32}\left(\frac{H}{2 h N}-\frac{4 \gamma_{2}}{\gamma_{1}}\right)_{+}^{2}\right\}
$$


where we have used $\frac{H}{h}-1 \geq \frac{H}{2 h}$ and $\gamma_{2} t \leq \frac{4 \gamma_{2}}{\gamma_{1}} N$. Collecting our bounds on the right-hand side of (1.23), we see that when (1.24) holds

$$
P_{x, \omega}\left[T_{x \cdot e_{2}+H}^{e_{2}} \leq T_{\widetilde{B}^{2}+x \cdot e_{2} e_{2}}\right] \leq \delta^{-1} \text {. }
$$

Using this to bound the inner probability in the rightmost expression of (1.22), we obtain

$$
P_{0, \omega}\left[T_{M 4 H}^{e_{2}}<T_{B}\right] \leq P_{0, \omega}\left[T_{(M-1) 4 H}^{e_{2}}<T_{B}\right] \cdot \delta^{-Z_{M-1}\left(e_{2}\right)},
$$

with the notation for $k \geq 0,|e|=1$ with $e \cdot e_{1}=0$,

$$
Z_{k}(e)=1\{\text { for all } z \in B \text { with }|z \cdot e-4 k H|<H,
$$

$$
\left.\Delta(z, \omega) \cdot e_{1} \geq \gamma_{1} L, \sup _{j \geq 2}\left|\Delta(z, \omega) \cdot e_{j}\right| \leq \gamma_{2} h\right\} .
$$

By induction, we now see that:

$$
P_{0, \omega}\left[T_{M 4 H}^{e_{2}}<T_{B}\right] \leq \delta^{-\sum_{0}^{M-1} Z_{k}\left(e_{2}\right)} .
$$

Similar estimates hold for each term in the right-hand side of (1.21). Further, the variables $Z_{k}(e), k \geq 0$, for fixed $e$ are i.i.d., with success probability bigger than $p$ [cf. (1.12)]. Hence by (1.21), (1.23) and (1.28),

$$
\begin{aligned}
\mathbb{P}\left[g^{c}\right] & =\mathbb{P}\left[P_{0, \omega}\left[\widetilde{T} \leq \widetilde{T}_{-N L} \wedge T_{N L}\right]>\kappa^{6 N L}\right] \\
& \leq \sum_{|e|=1, e \cdot e_{1}=0} \mathbb{P}\left[\delta^{-\sum_{0}^{M-1} Z_{k}(e)} \geq \frac{\kappa^{6 N L}}{2(d-1)}\right]
\end{aligned}
$$

and since $\frac{1}{2(d-1)} \kappa^{6 N L} \geq \kappa^{7 N L}$, using Azuma's inequality,

$$
\leq 2 d \exp \left\{-\frac{1}{2 M}\left(M p-7 N L \frac{\log \kappa^{-1}}{\log \delta}\right)_{+}^{2}\right\} .
$$

This and (1.18) complete the proof of (1.15).

We can now apply Theorem 1.1 to the derivation of a bound on $\mathbb{E}\left[\rho_{B_{0}}^{a}\right]$ [cf. (0.13)], with the choice

$$
B_{0}=B+2 e_{1}
$$

Corollary $1.3[d \geq 2$, under (0.1)]. Assume $0<a \leq 1$, and (1.14) holds, then

$$
\begin{aligned}
& \mathbb{E}\left[\rho_{B_{0}}^{a}\right] \leq \kappa^{-4 a} \mathbb{E}\left[\rho_{B}^{a}\right] \\
& \leq \kappa^{-4 a}\left(\frac{2 \mathbb{E}\left[\widehat{\rho}(0)^{2 a}\right]^{N / 2}}{\left(1-\mathbb{E}\left[\widehat{\rho}(0)^{2 a}\right]^{1 / 2}\right)_{+}}\right. \\
&\left.\quad+2 d \kappa^{-a N L} \exp \left\{-\frac{M}{2}\left(p-\frac{7 N L}{M} \frac{\log \kappa^{-1}}{\log \delta}\right)_{+}^{2}\right\}\right) .
\end{aligned}
$$


PROOF. By translation invariance,

$$
\mathbb{E}\left[\rho_{B_{0}}^{a}\right]=\mathbb{E}\left[\left(\frac{P_{-2 e_{1}, \omega}\left[X_{T_{B}} \notin \partial_{+} B\right]}{P_{-2 e_{1}, \omega}\left[X_{T_{B}} \in \partial_{+} B\right]}\right)^{a}\right],
$$

provided $\partial_{+} B$ is defined analogously as in (0.14) via

$$
\partial_{+} B=\left\{z \in \partial B, z \cdot e_{1} \geq N L, \sup _{i \geq 2}\left|z \cdot e_{i}\right|<\frac{1}{4}(N L)^{3}\right\} .
$$

Thanks to $(0.1)$, we have

$$
\kappa^{2} P_{0, \omega}\left[X_{T_{B}} \notin \partial_{+} B\right] \leq P_{-2 e_{1}, \omega}\left[X_{T_{B}} \notin \partial_{+} B\right] \leq \kappa^{-2} P_{0, \omega}\left[X_{T_{B}} \notin \partial_{+} B\right]
$$

and similar inequalities hold with $\left\{X_{T_{B}} \notin \partial_{+} B\right\}$ replaced by $\left\{X_{T_{B}} \in \partial_{+} B\right\}$. Hence we find

$$
\mathbb{E}\left[\rho_{B_{0}}^{a}\right] \leq \kappa^{-4 a} \mathbb{E}\left[\rho_{B}^{a}\right],
$$

and (1.31) follows from (1.15).

2. Deterministic bounds on Green functions $(\boldsymbol{d} \geq 3)$. The purpose of this section is to collect several controls on the Green function in a slab $U$ of thickness $2 L$ orthogonal to the direction $e_{1}$ for the random walk in an arbitrary environment $\omega \in \Omega_{\varepsilon}$; see below (0.7). Our main interest lies in the situation where $L$ is comparable to a small multiple of $\varepsilon^{-1}$. In this regime the random walk in an environment $\omega \in \Omega_{\varepsilon}$, killed when exiting $U$, will be a not too drastic perturbation of the simple random walk killed when exiting $U$. The estimates on Green functions derived in this section will hold uniformly for $\omega \in \Omega_{\varepsilon}$, and will be instrumental in Sections 3 and 4. We will obtain both $L^{p}$ and pointwise bounds. We first introduce some more notations. For $\omega \in \Omega_{\varepsilon}, 0<\varepsilon<1$, we write for $x \in \mathbb{Z}^{d}$, $|e|=1$,

$$
\omega(x, e)=\frac{1}{2 d}+\delta(x, e)
$$

and in view of (0.7),

$$
|\delta(x, e)| \leq \frac{\varepsilon}{4 d} .
$$

We recall that $\kappa$ is chosen equal to $(4 d)^{-1}$. The respective transition kernels of the walk in the environment $\omega \in \Omega_{\varepsilon}$ and of the simple random walk are

$$
R f(x)=\sum_{|e|=1} \omega(x, e) f(x+e), \quad R_{0} f(x)=\sum_{|e|=1} \frac{1}{2 d} f(x+e),
$$

where $f$ is some arbitrary real-valued function on $\mathbb{Z}^{d}$, and $x \in \mathbb{Z}^{d}$. For the time being, $L \geq 2$ is some integer, but we shall soon restrict $L$ to be smaller than a suitable multiple of $\varepsilon^{-1}$. We are interested in the slab $U$ introduced in (0.16),

$$
U=\left\{y \in \mathbb{Z}^{d},\left|y \cdot e_{1}\right|<L\right\} .
$$


We introduce the Green operators of the walks killed when exiting $U$,

$$
G_{U}=\sum_{n \geq 0}\left(\mathbb{1}_{U} R \mathbb{1}_{U}\right)^{n}, \quad G_{0, U}=\sum_{n \geq 0}\left(\mathbb{1}_{U} R_{0} \mathbb{1}_{U}\right)^{n},
$$

which are easily seen to define bounded operators on the space $L^{\infty}\left(\mathbb{Z}^{d}\right)$ of bounded functions endowed with the supremum norm. This space will contain all functions of interest in the present context. Before getting any further, it may be helpful to point out that our main object (cf. Section 4) is to show that the expectation of the variable $G_{U}\left(d \cdot e_{1}\right)(0)$ [ $=D$ in the notation of $\left.(0.15)\right]$ is positive and "dominates fluctuations." The deterministic bounds of this section will be helpful for this purpose.

The Green functions are defined via

$$
\begin{aligned}
g_{U}(x, y, \omega) & =\left(G_{U} \mathbb{1}_{y}\right)(x), \\
g_{0, U}(x, y) & =\left(G_{0, U} \mathbb{1}_{y}\right)(x), \quad x, y \in \mathbb{Z}^{d}, \omega \in \Omega_{\varepsilon}
\end{aligned}
$$

[the notation is coherent with (0.17)]. From the method of images (see also (3.15) of [18]), we have for $x, y \in U \cup \partial U$,

$$
g_{0, U}(x, y)=\sum_{k \in \mathbb{Z}}\left(g^{0}\left(x-y+2 k 2 L e_{1}\right)-g^{0}\left(x-y_{*}+(2 k+1) 2 L e_{1}\right)\right)
$$

provided $y_{*}$ stands for the image of $y$ under the reflection with respect to the hyperplane $\left\{z: z \cdot e_{1}=0\right\}$, and $g^{0}(\cdot)$ is the full space Green function of the simple random walk with pole at 0 (recall $d \geq 3$ ). The fact that

$$
\sum_{|e|=1} \delta(x, e)=0
$$

plays an important role in the sequel. In particular it implies that for any numerical function $f$ on $\mathbb{Z}^{d}, x \in \mathbb{Z}^{d}$,

$$
\left(R-R_{0}\right) f(x)=\sum_{|e|=1} \delta(x, e) f(x+e)=\sum_{|e|=1} \delta(x, e)(f(x+e)-f(x)) .
$$

This will be very helpful when comparing $G_{U}$ and $G_{0, U}$ with the usual perturbation identities

$$
\begin{aligned}
G_{U} & =G_{0, U}+G_{U} \mathbb{1}_{U}\left(R-R_{0}\right) \mathbb{1}_{U} G_{0, U} \\
& =G_{0, U}+G_{0, U} \mathbb{1}_{U}\left(R-R_{0}\right) \mathbb{1}_{U} G_{U} .
\end{aligned}
$$

We first gather some estimates on $g_{0, U}(\cdot, \cdot)$.

LEMMA 2.1.

$$
\sup _{x \in U} \sum_{y \in U} g_{0, U}(x, y) \leq c_{1} L^{2}=\sum_{y \in U} g_{0, U}(0, y) \quad \text { with } c_{1}=d,
$$




$$
\begin{aligned}
& \sup _{x \in U,|e|=1} \sum_{y \in U}\left(\left|g_{0, U}(x+e, y)-g_{0, U}(x, y)\right|\right. \\
& \left.\quad+\left|g_{0, U}(x, y+e)-g_{0, U}(x, y)\right|\right) \\
& \leq \frac{c_{2}(d)}{2} L
\end{aligned}
$$

$$
\begin{aligned}
\sup _{x \in U,|e|=1} \sum_{y \in U}[ & \left(g_{0, U}(x+e, y)-g_{0, U}(x, y)\right)^{2} \\
& \left.+\left(g_{0, U}(x, y+e)-g_{0, U}(x, y)\right)^{2}\right]
\end{aligned}
$$

$$
\leq c_{3}(d)
$$

(2.13) $\quad g_{0, U}(x, y) \leq \frac{c_{4}(d)}{(1+|x-y|)^{d-2}} \exp \left\{-\frac{c_{5}(d)}{L}\left|(x-y)_{\perp}\right|\right\}, \quad x, y \in U$,

with $z_{\perp}$ denoting the projection of $z$ on the orthogonal of $e_{1}$.

ProOF. We begin with (2.10). We denote by $Q_{x}$ the law of the simple random walk starting in $x$ and by $E^{Q_{x}}$ the corresponding expectation. Observe that under $Q_{x}, x \in \mathbb{Z}^{d}$,

$$
\left(X_{n} \cdot e_{1}\right)^{2}-\left(X_{0} \cdot e_{1}\right)^{2}-\frac{n}{d}, \quad n \geq 0 \text { is a martingale. }
$$

For $x \in U$, applying the stopping theorem, with the help of routine arguments, we find:

$$
\frac{1}{d} E^{Q_{x}}\left[T_{U}\right]=L^{2}-\left(x \cdot e_{1}\right)^{2} .
$$

The claim (2.10) easily follows since $E^{Q_{x}}\left[T_{U}\right]=\sum_{y} g_{0, U}(x, y)$. Let us now prove (2.13). For $x, y \in U$,

$$
g_{0, U}\left(x, X_{n \wedge T_{U} \wedge H_{x}}\right), \quad n \geq 0 \text { is a bounded martingale under } Q_{y}
$$

(we use the notation $H_{x}$ as a shorthand for $H_{\{x\}}$ [see (1.2)]). If we define $T=$ $\inf \left\{n \geq 0,\left|\left(X_{n}-y\right)_{\perp}\right| \geq \frac{1}{2}\left|(x-y)_{\perp}\right|\right\} \leq H_{x}, Q_{y}$-a.s., the stopping theorem yields

$$
\begin{aligned}
g_{0, U}(x, y) & =E^{Q_{y}}\left[g_{0, U}\left(x, X_{T_{U} \wedge T}\right)\right]=E^{Q_{y}}\left[g_{0, U}\left(x, X_{T}\right), T<T_{U}\right] \\
& \leq \sup \left\{g_{0, U}(x, z) ;\left|(z-x)_{\perp}\right| \geq \frac{1}{2}\left|(x-y)_{\perp}\right|-1\right\} Q_{y}\left[T<T_{U}\right] .
\end{aligned}
$$

Considering successive displacements of the simple random walk at $\left|(\cdot)_{\perp}\right|$-distance $4 L$, during which the walk has a nondegenerate probability of exiting $U$, it follows by standard arguments that

$$
Q_{y}\left[T<T_{U}\right] \leq c(d) \exp \left\{-\frac{c^{\prime}(d)}{L}\left|(x-y)_{\perp}\right|\right\} \quad \text { for } x, y \in U .
$$


On the other hand, we know from page 31 of Lawler [11] that

$$
g_{0, U}(x, y) \leq g^{0}(x-y) \leq \frac{c(d)}{(1+|x-y|)^{d-2}}, \quad x, y \in \mathbb{Z}^{d} .
$$

Combining (2.16)-(2.18), and considering the cases $\left|(x-y)_{\perp}\right| \leq L$ and $\left|(x-y)_{\perp}\right|>L$ separately, the claim (2.13) follows. As for (2.11) and (2.12), they are proved in the Appendix.

For $1 \leq p, q \leq \infty$, we denote by $|\cdot|_{p}$ the $L^{p}\left(\mathbb{Z}^{d}\right)$-norm and by $\|\cdot\|_{p, q}$ the operator norm from $L^{p}\left(\mathbb{Z}^{d}\right)$ into $L^{q}\left(\mathbb{Z}^{d}\right)$. We also write

$$
\nabla_{y} f(x)=f(x+y)-f(x),
$$

for $x, y \in \mathbb{Z}^{d}$ and $f$ a numerical function on $\mathbb{Z}^{d}$. We have the following controls on the norms of $G_{U}$ and $G_{0, U}$ :

Proposition 2.2. Assume that

$$
\varepsilon c_{1} L<\frac{3}{4} d, \quad \varepsilon c_{2} L<\frac{1}{2},
$$

then for $1 \leq p, q \leq \infty,|e|=1, \omega \in \Omega_{\varepsilon}$,

$$
\begin{gathered}
\left\|G_{U}\right\|_{p, q} \leq \frac{4}{3}\left\|G_{0, U}\right\|_{p, q}, \quad\left\|\nabla_{e} G_{U}\right\|_{p, q} \leq \frac{4}{3}\left\|\nabla_{e} G_{0, U}\right\|_{p, q}, \\
\left\|G_{0, U}\right\|_{p, p} \leq c_{1} L^{2}, \quad\left\|\nabla_{e} G_{0, U}\right\|_{p, p} \leq c_{2} L .
\end{gathered}
$$

Moreover, for a suitable $c_{6}(d) \in(0,1)$ :

$$
\sup _{x \in \mathbb{Z}^{d}, \omega \in \Omega_{\varepsilon}} E_{x, \omega}\left[\exp \left\{\frac{c_{6}}{L^{2}} T_{U}\right\}\right] \leq 2 .
$$

PROOF. We begin with (2.21). For $f$ a bounded function on $\mathbb{Z}^{d}$, and $x \in U$,

$$
\begin{aligned}
{\left[\mathbb{1}_{U}\right.} & \left.\left(R-R_{0}\right) \mathbb{1}_{U} G_{0, U} f\right](x) \\
\quad & =\left[\left(R-R_{0}\right) G_{0, U} f\right](x) \\
& \stackrel{(2.8)}{=} \sum_{|e|=1, y \in U} \delta(x, e)\left(g_{0, U}(x+e, y)-g_{0, U}(x, y)\right) f(y) .
\end{aligned}
$$

Hence, we find

$$
\left|\mathbb{1}_{U}\left(R-R_{0}\right) \mathbb{1}_{U} G_{0, U} f\right|_{\infty}
$$

$$
\begin{aligned}
& \stackrel{(2.2)}{\leq} \frac{\varepsilon}{4 d} \sup _{x \in U \cup \partial U} \sum_{|e|=1, y \in U}\left|g_{0, U}(x+e, y)-g_{0, U}(x, y) \| f\right|_{\infty} \\
& \stackrel{(2.11)}{\leq} \frac{\varepsilon}{2} c_{2} L|f|_{\infty} .
\end{aligned}
$$


(Note that when $x$ and $x+e$ belong to $\partial U$ the expression in the sum of the righthand side of the first inequality vanishes. To bound the terms where $x \in \partial U$, we hence only need consider the case $x \in \partial U, x+e \in U$, and writing $x+e=x^{\prime}$, $x=x^{\prime}+e^{\prime}$, we observe that we can replace $U \cup \partial U$ by $U$ in the supremum, and even $\frac{\varepsilon}{2}$ by $\frac{\varepsilon}{4}$ in the last inequality.) Similarly,

$$
\begin{aligned}
\mid \mathbb{1}_{U}( & \left.R-R_{0}\right)\left.\mathbb{1}_{U} G_{0, U} f\right|_{1} \\
& \leq \frac{\varepsilon}{4 d} \sup _{y \in U} \sum_{|e|=1, x \in U \cup \partial U}\left|g_{0, U}(x+e, y)-g_{0, U}(x, y)\right||f|_{1} \\
& \leq \frac{\varepsilon}{2} c_{2} L|f|_{1},
\end{aligned}
$$

where we have used (2.11), the symmetry of $g_{0, U}(\cdot, \cdot)$ (cf. [11], page 34) and taken into acount when $e=e_{1}$ or $-e_{1}$ the contribution of terms $x \in \partial U$, coming from "half of the boundary." It then follows from the Riesz-Thorin theorem (cf. Reed and Simon [16], page 27) that for $1 \leq p \leq \infty$,

$$
\left\|\mathbb{1}_{U}\left(R-R_{0}\right) \mathbb{1}_{U} G_{0, U}\right\|_{p, p} \leq \frac{\varepsilon}{2} c_{2} L \stackrel{(2.20)}{<} \frac{1}{4} .
$$

As a result $I-\mathbb{1}_{U}\left(R-R_{0}\right) \mathbb{1}_{U} G_{0, U}$ defines a bounded invertible operator from $L^{p}\left(\mathbb{Z}^{d}\right)$ into $L^{p}\left(\mathbb{Z}^{d}\right)$ and the norm of its inverse is smaller than $\frac{4}{3}$. From the first line of (2.9) we easily deduce (2.21). Let us turn to the proof of (2.22). From (2.10) and (2.11), we find by analogous considerations that for $p=1$ or $\infty$ and $|e|=1$,

$$
\left\|G_{0, U}\right\|_{p, p} \leq c_{1} L^{2}, \quad\left\|\nabla_{e} G_{0, U}\right\|_{p, p} \leq c_{2} L .
$$

The claim (2.22) then follows from the Riesz-Thorin theorem.

Let us finally prove (2.23). Observe that (2.21) and (2.22) imply that for $\omega \in \Omega_{\varepsilon}$,

$$
\sup _{x} E_{x, \omega}\left[T_{U}\right] \leq \frac{4}{3} c_{1} L^{2} \text {. }
$$

The claim now follows by a classical argument of Khas'minskii [8]; see, for instance, the proof of Lemma 1.1 of [18].

We will now derive pointwise bounds on $g_{U}$ in terms of $g_{0, U}$ which will be useful to control the fluctuations of $G_{U}\left(d \cdot e_{1}\right)(0)$.

Proposition 2.3. Let $\alpha \in[0,1)$, and assume

$$
\varepsilon L<\frac{1}{2} \frac{1-\alpha}{2-\alpha} \sqrt{c}_{6}
$$

then

$$
g_{U}(x, y, \omega) \leq c_{7}(d) g_{0, U}(x, y)^{1 /(2-\alpha)}, \quad x, y \in U, \omega \in \Omega_{\varepsilon}
$$


ProOF. For any $x \in \mathbb{Z}^{d}, n \geq 0, \omega \in \Omega_{\varepsilon}$, the law $P_{x, \omega}$ restricted to $\mathcal{F}_{n}$ is absolutely continuous with respect to $Q_{x}$, and

$$
P_{x, \omega}=Z_{n} Q_{x} \quad \text { on } \mathscr{F}_{n},
$$

provided

$$
Z_{n}=\exp \left\{\sum_{0}^{n-1} \log \left(1+2 d \delta\left(X_{k}, X_{k+1}-X_{k}\right)\right)\right\} .
$$

As a result of Taylor's formula with integral remainder for $|u| \leq \frac{1}{2}$,

$$
\log (1+u)=u-u^{2} \int_{0}^{1} \int_{0}^{v} \frac{1}{(1+u w)^{2}} d w d v .
$$

We now derive some integrability properties of $Z_{T_{U}}$ under $Q_{x}$. For $0<\beta<\varepsilon^{-1}$, $y \in \mathbb{Z}^{d}$, we have

$$
\begin{aligned}
& \frac{1}{2 d} \sum_{|e|=1} \exp \{\beta \log (1+\left.2 d \delta(y, e))-\beta^{2} \varepsilon^{2}\right\} \\
& \stackrel{(2.33)}{=} \frac{1}{2 d} \sum_{|e|=1} \exp \left\{\beta 2 d \delta(y, e)-\beta(2 d \delta(y, e))^{2}\right. \\
&\left.\times \int_{0}^{1} \int_{0}^{v} \frac{1}{(1+2 d \delta(y, e) w)^{2}} d w d v-\beta^{2} \varepsilon^{2}\right\} .
\end{aligned}
$$

But for each $|e|=1$, in view of (2.2),

$$
\beta^{2} \varepsilon^{2}=\beta^{2} \varepsilon^{2} \int_{0}^{1} \int_{0}^{v} 2 d w d v \geq(\beta 2 d \delta(y, e))^{2} \int_{0}^{1} \int_{0}^{v} \frac{1}{(1+\beta 2 d \delta(y, e) w)^{2}} d w d v
$$

hence coming back to the last line of (2.34), we obtain an upper bound by dropping the term containing the integral and replacing $\beta^{2} \varepsilon^{2}$ by the above lower bound. Using (2.33) again we see that

$$
\begin{aligned}
& \frac{1}{2 d} \sum_{|e|=1} \exp \left\{\beta \log (1+2 d \delta(y, e))-\beta^{2} \varepsilon^{2}\right\} \\
& \quad \leq \frac{1}{2 d} \sum_{|e|=1} \exp \{\log (1+\beta 2 d \delta(y, e))\}=1+\beta \sum_{|e|=1} \delta(y, e) \stackrel{(2.7)}{=} 1 .
\end{aligned}
$$

As a result we see that for $0<\beta<\varepsilon^{-1}$ and any $x$,

(2.36) $Z_{n}^{\beta} \exp \left\{-\beta^{2} \varepsilon^{2} n\right\}, \quad n \geq 0$, is an $\left(\mathcal{F}_{n}\right)$-supermartingale under $Q_{x}$.

In particular if $0<2 \beta \varepsilon L<\sqrt{c}_{6}$, then $2 \beta \varepsilon<1$, and for any $x \in U$,

$$
\begin{gathered}
E^{Q_{x}}\left[Z_{T_{U}}^{\beta}\right] \leq E^{Q_{x}}\left[Z_{T_{U}}^{2 \beta} \exp \left\{-4 \beta^{2} \varepsilon^{2} T_{U}\right\}\right]^{1 / 2} E^{Q_{x}}\left[\exp \left\{4 \beta^{2} \varepsilon^{2} T_{U}\right\}\right]^{1 / 2} \\
\stackrel{(2.36)}{\leq} E^{Q_{x}}\left[\exp \left\{4 \beta^{2} \varepsilon^{2} T_{U}\right\}\right]^{1 / 2} \stackrel{(2.23)}{\leq} \sqrt{2}
\end{gathered}
$$


[Note that each $\Omega_{\varepsilon^{\prime}}, 0<\varepsilon^{\prime}<1$, contains the constant environment corresponding to the simple random walk so that (2.23) applies to $Q_{x}$.] We can choose

$$
\beta=\frac{2-\alpha}{1-\alpha}, \quad \beta^{\prime}=2-\alpha \quad\left(\text { so that } \frac{1}{\beta}+\frac{1}{\beta^{\prime}}=1, \beta \geq 2 \geq \beta^{\prime}>1\right),
$$

and in view of (2.29), $0<2 \beta \varepsilon L<\sqrt{c}_{6}$. Then for $x, y \in U, \omega \in \Omega_{\varepsilon}$,

$$
g_{U}(x, y, \omega)=\lim _{n \rightarrow \infty} E_{x, \omega}\left[\sum_{0}^{T_{U} \wedge n-1} \mathbb{1}_{y}\left(X_{k}\right)\right]
$$

using (2.31), the martingale property of $Z_{n}$ and then the uniform integrability stemming from (2.37) and (2.23),

$$
\begin{aligned}
& \quad=\lim _{n} E^{Q_{x}}\left[\left(\sum_{0}^{T_{U} \wedge n-1} \mathbb{1}_{y}\left(X_{k}\right)\right) Z_{T_{U} \wedge n}\right]=E^{Q_{x}}\left[\left(\sum_{0}^{T_{U}-1} \mathbb{1}_{y}\left(X_{k}\right)\right) Z_{T_{U}}\right] \\
& \stackrel{\text { Hölder }}{\leq} E^{Q_{x}}\left[\left(\sum_{0}^{T_{U}-1} \mathbb{1}_{y}\left(X_{k}\right)\right)^{\beta^{\prime}}\right]^{1 / \beta^{\prime}} E^{Q_{x}}\left[Z_{T_{U}}^{\beta}\right]^{1 / \beta} .
\end{aligned}
$$

Applying once more Hölder's inequality on the first term since $\beta^{\prime}=\alpha+2(1-\alpha)$, and using (2.37) to control the second term,

$$
\leq E^{Q_{x}}\left[\sum_{0}^{T_{U}-1} \mathbb{1}_{y}\left(X_{k}\right)\right]^{\alpha / \beta^{\prime}} E^{Q_{x}}\left[\left(\sum_{0}^{T_{U}-1} \mathbb{1}_{y}\left(X_{k}\right)\right)^{2}\right]^{(1-\alpha) / \beta^{\prime}} 2^{1 / 2 \beta} .
$$

Observe now that

$$
\begin{aligned}
E^{Q_{x}}\left[\left(\sum_{0}^{T_{U}-1} \mathbb{1}_{y}\left(X_{k}\right)\right)^{2}\right] & \leq 2 E^{Q_{x}}\left[\sum_{0 \leq k \leq m<T_{U}} \mathbb{1}_{y}\left(X_{k}\right) \mathbb{1}_{y}\left(X_{m}\right)\right] \\
& =2 g_{0, U}(x, y) g_{0, U}(y, y) .
\end{aligned}
$$

Since $g_{0, U}(y, y) \leq g^{0}(0), \frac{1-\alpha}{\beta^{\prime}} \leq 1,2 \beta \geq 1$, (2.30) follows from the last line of (2.39) and (2.40).

As a slight variation of (2.36), we mention the following estimate which will be used in Section 3.

Lemma 2.4. When $2 \varepsilon L<\sqrt{c}_{6}$, then in the notation of (2.32),

$$
E^{Q_{x}}\left[Z_{T_{U}}^{-1}\right] \leq \sqrt{2} \quad \text { for any } x .
$$


Proof. As in (2.34), for $y \in \mathbb{Z}^{d},|e|=1$,

$$
\begin{aligned}
& \frac{1}{2 d} \sum_{|e|=1} \exp \{-2 \log (1\left.+2 d \delta(y, e))-4 \varepsilon^{2}\right\} \\
&=\frac{1}{2 d} \sum_{|e|=1} \exp \{-4 d \delta(y, e)+2(2 d \delta(y, e))^{2} \\
&\left.\times \int_{0}^{1} \int_{0}^{v} \frac{1}{(1+2 d \delta(y, e) w)^{2}} d w d v-4 \varepsilon^{2}\right\}
\end{aligned}
$$

but $\varepsilon<\frac{1}{2}$ [indeed, $c_{6} \in(0,1)$; cf. (2.31)] and

$$
\begin{aligned}
4 \varepsilon^{2}=2 \varepsilon^{2} \int_{0}^{1} \int_{0}^{v} 4 d w d v & \\
\geq(4 d \delta(y, e))^{2} & {\left[\int_{0}^{1} \int_{0}^{v} \frac{1}{(1+2 d \delta(y, e) w)^{2}} d w d v\right.} \\
& \left.+\int_{0}^{1} \int_{0}^{v} \frac{1}{(1-4 d \delta(y, e) w)^{2}} d w d v\right],
\end{aligned}
$$

and the last member of (2.42) is smaller than

$$
\begin{aligned}
\sum_{e} \frac{1}{2 d} \exp \{ & -4 d \delta(y, e) \\
& \left.-(4 d \delta(y, e))^{2} \int_{0}^{t} \int_{0}^{v} \frac{1}{(1-4 d \delta(y, e) w)^{2}} d w d v\right\} \stackrel{(2.7),(2.33)}{=} 1 .
\end{aligned}
$$

We hence see from Cauchy-Schwarz's inequality that

$$
{ }_{E}{ }^{Q_{x}}\left[Z_{T_{U}}^{-1}\right] \leq E^{Q_{x}}\left[Z_{T_{U}}^{-2} e^{-4 \varepsilon^{2} T_{U}}\right]^{1 / 2} E^{Q_{x}}\left[e^{4 \varepsilon^{2} T_{U}}\right]^{1 / 2} \leq \sqrt{2}
$$

by a similar argument as in (2.37) and by (2.23).

3. Stochastic bounds on exit measures $(d \geq 3)$. The main purpose of this section is to control the random variable $\widehat{p}(0, \omega)$ [cf. (1.6)], which describes the probability that the walk starting at the origin exits the slab $U$ [cf. (0.16)], "to the right." Throughout this section we assume that the single site distribution $\mu$ is concentrated on $\wp_{\varepsilon}$ [cf. (0.7)]. The random variable $\widehat{p}(0, \omega)$ is closely related to the random variable $G_{U}\left(d \cdot e_{1}\right)(0)$ [ $=D$ in the notations of $\left.(0.15)\right]$. Indeed, it follows from the stopping theorem applied to the $P_{x, \omega}$-martingale $X_{n} \cdot e_{1}-\sum_{0}^{n-1} d\left(X_{k}, \omega\right) \cdot e_{1}$ that for $0<\varepsilon<1, L \geq 1$ integer, $\omega \in \Omega_{\varepsilon}$,

$$
\widehat{p}(x, \omega)=\frac{1}{2}+\frac{1}{2 L} G_{U}\left(d \cdot e_{1}\right)(x) \quad \text { for } x \in \mathbb{Z}^{d} \text { with } x \cdot e_{1}=0 .
$$

We want to show in this section that when $L$ is comparable to a small multiple of $\varepsilon$, and $\varepsilon$ is small but $\lambda=\mathbb{E}\left[d(0, \omega) \cdot e_{1}\right]$ is not too small in the sense of $(0.9)$, then the 
expectation of $G_{U}\left(d \cdot e_{1}\right)(0)$ is positive and dominates the "relevant fluctuations" of $G_{U}\left(d \cdot e_{1}\right)(0)$. We begin with a lower bound on $\mathbb{E}\left[G_{U}\left(d \cdot e_{1}\right)(0)\right]$. A special cancellation in the term $B$ of (3.14) will play an important role in the derivation of this lower bound.

Proposition 3.1. Assume $0<8 d \varepsilon<1$, and $L \geq 2$ is an integer.

Let (2.20) and the assumptions of Lemma 2.4 hold. If

$$
\lambda=\mathbb{E}\left[d(0, \omega) \cdot e_{1}\right]>c_{8}(d) \varepsilon^{2}\left(\varepsilon \log L+\frac{1}{L}\right),
$$

then

$$
\mathbb{E}\left[G_{U}\left(d \cdot e_{1}\right)(0)\right] \geq \frac{2}{5} c_{1} \lambda L^{2}
$$

ProOF. We write for $x \in \mathbb{Z}^{d},|e|=1, \omega \in \Omega_{\varepsilon}$,

$$
\begin{gathered}
\tilde{\delta}(x, e)=\delta(x, e)-\mathbb{E}[\delta(x, e)], \\
\tilde{d}(x, \omega)=d(x, \omega)-\mathbb{E}[d(x, \omega)] .
\end{gathered}
$$

Observe that

$$
G_{U}\left(d \cdot e_{1}\right)(0)=\lambda\left(G_{U} \mathbb{1}\right)(0)+G_{U}\left(\tilde{d} \cdot e_{1}\right)(0) .
$$

In view of (2.20), (2.25) and the first line of (2.9),

$$
\left(G_{U} \mathbb{1}\right)(0) \geq\left(G_{0, U} \mathbb{1}-\frac{1}{4} G_{U} \mathbb{1}\right)(0),
$$

so that for $\omega \in \Omega_{\varepsilon}$,

$$
\left(G_{U} \mathbb{1}\right)(0) \geq \frac{4}{5}\left(G_{0, U} \mathbb{1}\right)(0) \stackrel{(2.10)}{=} \frac{4}{5} c_{1} L^{2} .
$$

Hence our claim will follow once we show that

$$
\left|\mathbb{E}\left[G_{U}\left(\tilde{d} \cdot e_{1}\right)(0)\right]\right| \leq \frac{2}{5} c_{1} \lambda L^{2} .
$$

By a classical Markov chain calculation,

$$
G_{U}\left(\tilde{d} \cdot e_{1}\right)(0)=\sum_{x \in U} P_{0, \omega}\left[H_{x}<T_{U}\right] \frac{\tilde{d}(x) \cdot e_{1}}{P_{x, \omega}\left[\widetilde{H}_{x}>T_{U}\right]},
$$

provided for $z \in \mathbb{Z}^{d}, \widetilde{H}_{z}$ denotes the hitting time of $z$,

$$
\tilde{H}_{z}=\inf \left\{n \geq 1, X_{n}=z\right\} .
$$

Let us write for $x \in \mathbb{Z}^{d}, \omega \in \Omega_{\varepsilon}$,

$$
\begin{aligned}
& \bar{P}_{x, \omega}=P_{x, \bar{\omega}_{x}} \quad \text { where } \bar{\omega}_{x}(y, \cdot)=\omega(y, \cdot) \text {, when } y \neq x, \\
& \bar{\omega}_{x}(x, \cdot)=\mathbb{E}[\omega(0, \cdot)] .
\end{aligned}
$$


(Note that $\bar{\omega}_{x} \in \Omega_{\varepsilon}$.) We find

$$
\begin{aligned}
G_{U}\left(\tilde{d} \cdot e_{1}\right)(0)=\sum_{x \in U} & P_{0, \omega}\left[H_{x}<T_{U}\right]\left(\tilde{d}(x) \cdot e_{1}\right) \\
& \times\left(\bar{P}_{x, \omega}\left[\tilde{H}_{x}>T_{U}\right]+\sum_{|e|=1} \tilde{\delta}(x, e) P_{x+e, \omega}\left[H_{x}>T_{U}\right]\right)^{-1} \\
= & \sum_{x \in U} P_{0, \omega}\left[H_{x}<T_{U}\right] \frac{\tilde{d}(x) \cdot e_{1}}{\bar{P}_{x, \omega}\left[\widetilde{H}_{x}>T_{U}\right]} \\
& \times\left(1+\sum_{|e|=1} \tilde{\delta}(x, e) \frac{P_{x+e, \omega}\left[H_{x}>T_{U}\right]}{\bar{P}_{x, \omega}\left[\widetilde{H}_{x}>T_{U}\right]}\right)^{-1}
\end{aligned}
$$

From (0.1) (recall that here $\kappa=\frac{1}{4 d}$ ),

$$
P_{x+e, \omega}\left[H_{x}>T_{U}\right] \leq \kappa^{-1} \bar{P}_{x, \omega}\left[\tilde{H}_{x}>T_{U}\right] \quad \text { for } x \in U,|e|=1 .
$$

Hence from (2.2) and our assumption on $\varepsilon$,

$$
\left|\sum_{|e|=1} \tilde{\delta}(x, e) \frac{P_{x+e, \omega}\left[H_{x}>T_{U}\right]}{\bar{P}_{x, \omega}\left[\widetilde{H}_{x}>T_{U}\right]}\right| \leq \frac{\varepsilon}{\kappa} \leq \frac{1}{2} .
$$

However for $|u| \leq \frac{1}{2}$,

$$
\left|(1-u)^{-1}-1-u\right|=\frac{u^{2}}{1-u} \leq 2 u^{2},
$$

and as a result

$$
\begin{aligned}
& G_{U}\left(\tilde{d} \cdot e_{1}\right)(0)=A+B+C, \quad \text { where } \\
& A=\sum_{x \in U} P_{0, \omega}\left[H_{x}<T_{U}\right] \frac{\tilde{d}(x) \cdot e_{1}}{\bar{P}_{x, \omega}\left[\widetilde{H}_{x}>T_{U}\right]} \\
& B=-\sum_{x \in U} \frac{P_{0, \omega}\left[H_{x}<T_{U}\right]}{\bar{P}_{x, \omega}\left[\widetilde{H}_{x}>T_{U}\right]}\left(\tilde{d}(x) \cdot e_{1}\right) \sum_{|e|=1} \tilde{\delta}(x, e) \frac{P_{x+e, \omega}\left[H_{x}>T_{U}\right]}{\bar{P}_{x, \omega}\left[\widetilde{H}_{x}>T_{U}\right]},
\end{aligned}
$$

and by (3.12), (3.13) and (2.2),

$$
C \leq 2 \sum_{x \in U} \frac{P_{0, \omega}\left[H_{x}<T_{U}\right]}{\bar{P}_{x, \omega}\left[\widetilde{H}_{x}>T_{U}\right]} \varepsilon\left(\frac{\varepsilon}{\kappa}\right)^{2} \leq 2\left(\frac{\varepsilon}{\kappa}\right)^{3}\left(G_{U} \mathbb{1}\right)(0) \stackrel{(2.21),(2.22)}{\leq} \frac{8}{3} c_{1}\left(\frac{\varepsilon}{\kappa}\right)^{3} L^{2}
$$

where we have used in the second inequality the fact that

$$
\bar{P}_{x, \omega}\left[\tilde{H}_{x}>T_{U}\right] \geq \kappa P_{x, \omega}\left[\tilde{H}_{x}>T_{U}\right] \quad \text { for } x \in \mathbb{Z}^{d}, \omega \in \Omega_{\varepsilon},
$$

and the analogue of (3.8) with $\mathbb{1}$ in place of $\tilde{d} \cdot e_{1}$. 
Observe at this point that $P_{0, \omega}\left[H_{x}<T_{U}\right]$ and $\bar{P}_{x, \omega}\left[\widetilde{H}_{x}>T_{U}\right]$ are independent of $\omega(x, \cdot)$ so that

$$
\mathbb{E}[A]=0 .
$$

By choosing $c_{8}(d)$ large enough in (3.2), we can make sure that $\frac{1}{5} c_{1} \lambda \geq$ $\frac{8}{3} c_{1}\left(\frac{\varepsilon}{\kappa}\right)^{3}$.

Hence (3.7) will follow once we show that

$$
|\mathbb{E}[B]| \leq \frac{1}{5} c_{1} \lambda L^{2} .
$$

This is where the special cancellation showing that "there is no term of order $\varepsilon^{2} L^{2}$ in $B$ " comes into play.

In view of (2.7), for $x \in \mathbb{Z}^{d}, \omega \in \Omega_{\varepsilon}$,

$$
\sum_{|e|=1} \tilde{\delta}(x, e)=0 .
$$

As a consequence, $B$ remains unchanged if the rightmost term $P_{x+e, \omega}\left[H_{x}>\right.$ $\left.T_{U}\right]$ is replaced by $P_{x+e, \omega}\left[H_{x}<T_{U}\right]-P_{x+e_{1}, \omega}\left[H_{x}<T_{U}\right]$. Note also that by similar arguments as in the derivation of (2.16) (replacing $T$ by $H_{x}$ ),

$$
P_{y, \omega}\left[H_{x}<T_{U}\right]=\frac{g_{U}(y, x, \omega)}{g_{U}(x, x, \omega)} \quad \text { for } x, y \in \mathbb{Z}^{d}, \omega \in \Omega_{\varepsilon} .
$$

As a result, using also (3.15), we obtain

$$
\begin{aligned}
& |B| \leq \frac{1}{\kappa^{2}} \sum_{x \in U} \frac{P_{0, \omega}\left[H_{x}<T_{U}\right]}{P_{x, \omega}\left[\widetilde{H}_{x}>T_{U}\right]^{2}}\left|\widetilde{d}(x) \cdot e_{1}\right| \\
& \times \sum_{|e|=1}|\widetilde{\delta}(x, e)|\left|g_{U}(x+e, x, \omega)-g_{U}\left(x+e_{1}, x, \omega\right)\right| \\
& \stackrel{(2.2)}{\leq}\left(\frac{\varepsilon}{\kappa}\right)^{2} \sup _{|e|=1} \sum_{x \in U} g_{U}(0, x, \omega) \mid g_{U}(x+e, x, \omega) \\
& -g_{U}\left(x+e_{1}, x, \omega\right) \mid P_{x, \omega}\left[\tilde{H}_{x}>T_{U}\right]^{-1} .
\end{aligned}
$$

Further, from the Cauchy-Schwarz inequality, Lemma 2.4 and (2.37) with $\beta \in\left(1, \frac{\sqrt{c_{6}}}{2 \varepsilon L}\right)$,

$$
\begin{aligned}
Q_{x}\left[\tilde{H}_{x}>T_{U}\right] & \leq E^{Q_{x}}\left[\tilde{H}_{x}>T_{U}, Z_{T_{U}}\right]^{1 / 2} E^{Q_{x}}\left[Z_{T_{U}}^{-1}\right]^{1 / 2} \\
& =P_{x, \omega}\left[\widetilde{H}_{x}>T_{U}\right]^{1 / 2} E^{Q_{x}}\left[Z_{T_{U}}^{-1}\right]^{1 / 2} \\
& \leq \sqrt{2} P_{x, \omega}\left[\widetilde{H}_{x}>T_{U}\right]^{1 / 2} .
\end{aligned}
$$

Since the left-hand side of (3.21) is bounded away from 0 by a dimension dependent constant (recall $d \geq 3$ ), the same holds true for $P_{x, \omega}\left[\widetilde{H}_{x}>T_{U}\right]$. Hence 
to prove (3.17) it suffices to show that for some $c_{9}(d)>0$, any $|e|=1, \omega \in \Omega_{\varepsilon}$, $L \geq 2$ integer,

$$
\begin{aligned}
& \sum_{x \in U} g_{U}(0, x, \omega)\left|g_{U}(x+e, x, \omega)-g_{U}\left(x+e_{1}, x, \omega\right)\right| \\
& \quad \leq c_{9}\left(\varepsilon \log L+\frac{1}{L}\right) L^{2} .
\end{aligned}
$$

Using the first line of (2.9), the left-hand side of (3.22) is smaller than the sum $D+E$, where

$$
D=\sum_{x \in U} g_{U}(0, x, \omega)\left|g_{0, U}(x+e, x)-g_{0, U}\left(x+e_{1}, x\right)\right|
$$

and

$$
E=2 \sup _{\left|e^{\prime}\right|=1} \sum_{x \in U} g_{U}(0, x, \omega)\left|\left(\nabla_{e^{\prime}} G_{U} \mathbb{1}_{U}\left(R-R_{0}\right) \mathbb{1}_{U} G_{0, U} \mathbb{1}_{x}\right)(x)\right| .
$$

We first bound $E$. To this end we note that

$$
\begin{aligned}
& \left|\left(\nabla_{e^{\prime}} G_{U} \mathbb{1}_{U}\left(R-R_{0}\right) \mathbb{1}_{U} G_{0, U} \mathbb{1}_{x}\right)(x)\right| \\
& \quad=\left|\sum_{y \in U,\left|e^{\prime \prime}\right|=1} \nabla_{e^{\prime}} g_{U}(x, y, \omega) \delta\left(y, e^{\prime \prime}\right) \nabla_{e^{\prime \prime}} g_{0, U}(y, x)\right| .
\end{aligned}
$$

Here $\nabla_{e^{\prime}}$ and $\nabla_{e^{\prime \prime}}$, respectively, operate on the first variable of the functions $g_{U}$ and $g_{0, U}$,

$$
\begin{aligned}
& \leq \varepsilon \sup _{x \in U,\left|e^{\prime \prime}\right|=1}\left(\sum_{y \in U}\left(\nabla_{e^{\prime}} g_{U}(x, y)\right)^{2}\right)^{1 / 2}\left(\sum_{y \in U}\left(\nabla_{e^{\prime \prime}} g_{0, U}(y, x)\right)^{2}\right)^{1 / 2} \\
& \stackrel{(2.12)}{\leq} \varepsilon \sqrt{c_{3}}\left\|\nabla_{e^{\prime}} G_{U}\right\|_{2, \infty} \stackrel{(2.21)}{\leq} \frac{4}{3} \varepsilon \sqrt{c_{3}}\left\|\nabla_{e^{\prime}} G_{0, U}\right\|_{2, \infty} \stackrel{(2.12)}{\leq} \frac{4}{3} \varepsilon c_{3} .
\end{aligned}
$$

We hence find with the help of (2.10) and (2.21),

$$
|E| \leq \frac{32}{9} c_{1} c_{3} \varepsilon L^{2} \text {. }
$$

We now bound $D$. From (2.6) and the identity $g^{0}(e)=g^{0}\left(e_{1}\right)$ for $|e|=1$, it follows that for $x \in U,|e|=1$,

$$
\begin{aligned}
& \left|g_{0, U}(x+e, x)-g_{0, U}\left(x+e_{1}, x\right)\right| \\
& \leq \sum_{k \neq 0}\left|g^{0}\left(e+2 k 2 L e_{1}\right)-g^{0}\left(e_{1}+2 k 2 L e_{1}\right)\right| \\
& \quad+\sum_{k \neq 0,-1}\left|\nabla_{e-e_{1}} g^{0}\left(e_{1}+2 x_{1} e_{1}+(2 k+1) 2 L e_{1}\right)\right| \\
& \quad+\left|\nabla_{e-e_{1}} g^{0}\left(e_{1}+2 x_{1} e_{1}+2 L e_{1}\right)\right|+\left|\nabla_{e-e_{1}} g^{0}\left(e_{1}+2 x_{1} e_{1}-2 L e_{1}\right)\right|,
\end{aligned}
$$

where $x_{1}$ denotes the first coordinate of $x$. Note that for $k \neq 0,-1$ and $m=2 k+1$,

$$
|m| \geq 3 \text { and }\left|2 x_{1}+1+(2 k+1) 2 L e_{1}\right| \geq|(|m|-1) 2 L-1| \geq(|m|-2) 2 L .
$$


From Theorem 1.5.5 of Lawler [10], the second sum in the right-hand side of (3.27) is smaller than

$$
c(d) \sum_{|m| \geq 3}(|m|-2)^{-(d-1)} L^{-(d-1)} \leq c^{\prime}(d) L^{-(d-1)} .
$$

A similar bound holds for the first sum on the right-hand side of (3.27). As a result we find that

$$
\begin{aligned}
& \left|g_{0, U}(x+e, x)-g_{0, U}\left(x+e_{1}, x\right)\right| \\
& \quad \leq c(d)\left(L^{1-d}+\left|L-x_{1}\right|^{1-d}+\left|L+x_{1}\right|^{1-d}\right) \quad \text { for } x \in U .
\end{aligned}
$$

Keeping (3.26) in mind, we see that the claim (3.22) will follow provided we can show a similar inequality with the term $\left|g_{U}(x+e, x, \omega)-g_{U}\left(x+e_{1}, x, \omega\right)\right|$ replaced by $\left|L-x_{1}\right|^{1-d}+\left|L+x_{1}\right|^{1-d}$. We therefore consider

$$
\begin{aligned}
& \sum_{x \in U} g_{U}(0, x, \omega)\left|L-x_{1}\right|^{1-d} \stackrel{(2.9)}{=} \sum_{x \in U} g_{0, U}(0, x)\left|L-x_{1}\right|^{1-d}+F \text { with } \\
& F=\sum_{x, y \in U,|e|=1} g_{U}(0, y, \omega) \delta(y, e) \nabla_{e} g_{0, U}(y, x)\left|L-x_{1}\right|^{1-d} .
\end{aligned}
$$

Using (A.23) from the Appendix, together with (2.10) and (2.21) we find

$$
\begin{aligned}
|F| \leq \varepsilon\left(G_{U} \mathbb{1}\right)(0) \sup _{y \in U} \sum_{x \in U} c(d)\left(L^{1-d}+\left(1+\left|(y-x)_{\perp}\right|\right)^{1-d}\right) \\
\quad \times \exp \left(-\frac{c^{\prime}(d)\left|(y-x)_{\perp}\right|}{L}\right)\left|L-x_{1}\right|^{1-d} \\
\leq c(d) \varepsilon L^{2} \sum_{w \in \mathbb{Z}^{d-1}}\left(L^{1-d}+(1+|w|)^{1-d}\right) \exp \left(-\frac{c^{\prime}(d)|w|}{L}\right) \sum_{k=1}^{2 L} k^{1-d} \\
\leq c(d) \varepsilon L^{2} \sum_{1}^{\infty} m^{d-2}\left(L^{1-d}+m^{1-d}\right) \exp \left(-\frac{c^{\prime}(d) m}{L}\right) \leq c(d) \varepsilon L^{2} \log L .
\end{aligned}
$$

On the other hand, we see from (2.13) that

$$
\begin{aligned}
& \sum_{x \in U} g_{0, U}(0, x)\left|L-x_{1}\right|^{1-d} \\
& \quad \leq \sum_{x \in U} c(d)(1+|x|)^{2-d} \exp \left(-\frac{c^{\prime}(d)\left|x_{\perp}\right|}{L}\right)\left|L-x_{1}\right|^{1-d}
\end{aligned}
$$

and by a similar argument as above,

$$
\leq c(d) \sum_{w \in \mathbb{Z}^{d-1}}(1+|w|)^{2-d} \exp \left(-\frac{c^{\prime}(d)|w|}{L}\right) \leq c(d) L .
$$

We have thus obtained that for $|e|=1, \omega \in \Omega_{\varepsilon}, L \geq 2$ integer,

$$
\sum_{x \in U} g(0, x, \omega)\left|L-x_{1}\right|^{1-d} \leq c_{10}(d)\left(\varepsilon \log L+\frac{1}{L}\right) L^{2} \text {. }
$$


A similar estimate naturally holds with $\left|L+x_{1}\right|^{1-d}$ in place of $\left|L-x_{1}\right|^{1-d}$. This finishes the proof of (3.22) and hence of Proposition 3.1.

We now turn to the control of fluctuations of $G_{U}\left(d \cdot e_{1}\right)(0)$. In the notation of (0.18), our main estimate comes in the following.

Proposition 3.2. Let $\alpha \in[0,1), \varepsilon \in(0,1), L \geq 2$ be an integer, and assume (2.20) and (2.29). Then for $u>0$,

$$
\mathbb{P}\left[\left|G_{U}\left(d \cdot e_{1}\right)(0)-\mathbb{E}\left[G_{U}\left(d \cdot e_{1}\right)(0)\right]\right|>u\right] \leq 2 \exp \left\{-\frac{u^{2}}{c_{\alpha, L}}\right\},
$$

where

$$
c_{\alpha, L}=c_{11}(d) \varepsilon^{2} \sum_{y \in U} g_{0, U}(0, y)^{2 /(2-\alpha)},
$$

and for some $c_{12}(\alpha, d)>0$,

$$
c_{\alpha, L} \leq \begin{cases}c_{12} \varepsilon^{2} L^{1+(2(1-\alpha) /(2-\alpha))}, & \text { when } d=3, \\ c_{12} \varepsilon^{2} L^{(4(1-\alpha) /(2-\alpha))}, & \text { when } d=4, \\ c_{12} \varepsilon^{2}, & \text { when } d \geq 5 \text { and } \alpha>\frac{4}{5} .\end{cases}
$$

PROOF. We use the martingale method. Namely, we introduce $x_{i}, i \geq 1$, an enumeration of $U$ and define the filtration

$$
g_{n}= \begin{cases}\sigma\left(\omega\left(x_{1}, \cdot\right), \ldots, \omega\left(x_{n}, \cdot\right)\right), & n \geq 1, \\ \{\phi, \Omega\}, & n=0,\end{cases}
$$

as well as the bounded $g_{n}$-martingale

$$
H_{n}=\mathbb{E}\left[G_{U}\left(d \cdot e_{1}\right)(0) \mid \mathscr{G}_{n}\right], \quad n \geq 0 .
$$

We will show that under the assumptions of Proposition 3.2,

$$
\left|H_{n}-H_{n-1}\right| \leq c_{13}(d) \varepsilon g_{0, U}\left(0, x_{n}\right)^{1 /(2-\alpha)} \stackrel{\text { def }}{=} \gamma_{n}, \quad n \geq 1 .
$$

Using a slight variation of the proof of Azuma's inequality (cf. [1], pages 240 and 234), it will follow that for $n \geq 1$ and $\rho \in \mathbb{R}$,

$$
\mathbb{E}\left[\exp \left\{\rho\left(H_{n}-H_{n-1}\right)\right\} \mid \mathscr{g}_{n-1}\right] \leq \exp \left\{\frac{1}{2} \rho^{2} \gamma_{n}^{2}\right\}
$$

from which it follows by routine arguments that

$$
\mathbb{E}\left[\exp \left\{\rho\left(G_{U}\left(d \cdot e_{1}\right)(0)-\mathbb{E}\left[G_{U}\left(d \cdot e_{1}\right)(0)\right]\right)\right\}\right] \leq \exp \left\{\frac{1}{2} \rho^{2} \sum_{n \geq 1} \gamma_{n}^{2}\right\}
$$


since $H_{0}=\mathbb{E}\left[G_{U}\left(d \cdot e_{1}\right)(0)\right]$ and $H_{\infty}=G_{U}\left(d \cdot e_{1}\right)(0)$. The claim (3.33) is then an immediate consequence and (3.35) readily follows from (2.13). Hence our main task is to prove (3.38). To this end, for $n \geq 1$, and $\omega, \omega^{\prime}$ in $\Omega_{\varepsilon}$ which coincide at each $x_{i}, i \neq n$, we define

$$
\Gamma_{n}\left(\omega, \omega^{\prime}\right)=G_{U}\left(d \cdot e_{1}\right)\left(0, \omega^{\prime}\right)-G_{U}\left(d \cdot e_{1}\right)(0, \omega) .
$$

Let us introduce $\omega_{u}\left(x_{i}, \cdot\right)=(1-u) \omega\left(x_{i}, \cdot\right)+u \omega^{\prime}\left(x_{i}, \cdot\right)$, for $i \geq 1, u \in[0,1]$. Notice that $\omega_{u}$ belongs to $\Omega_{\varepsilon}$ and coincides with $\omega$ and $\omega^{\prime}$ except maybe at $x_{n}$. Also by rather analogous considerations as in (3.8),

$$
\begin{aligned}
G_{U}\left(d \cdot e_{1}\right)\left(0, \omega_{u}\right)= & E_{0, \omega_{u}}\left[\sum_{0}^{H_{x_{n}} \wedge T_{U}-1}\left(d \cdot e_{1}\right)\left(X_{k}, \omega_{u}\right)\right] \\
& +\frac{P_{0, \omega_{u}}\left[H_{x_{n}}<T_{U}\right]}{P_{x_{n}, \omega_{u}}\left[\widetilde{H}_{x_{n}}>T_{U}\right]} E_{x_{n}, \omega_{u}}\left[\sum_{0}^{\left[\widetilde{H}_{x_{n}} \wedge T_{U}-1\right.}\left(d \cdot e_{1}\right)\left(X_{k}, \omega_{u}\right)\right] .
\end{aligned}
$$

Observe that neither $P_{0, \omega_{u}}\left[H_{x_{n}}<T_{U}\right]$ nor the first term on the right-hand side of (3.42) depend on $u$. Differentiating in the $u$ variable, we obtain

$$
\begin{aligned}
& \partial_{u} G_{u}\left(d \cdot e_{1}\right)\left(0, \omega_{u}\right) \\
& =\frac{P_{0, \omega_{u}}\left[H_{x_{n}}<T_{U}\right]}{P_{x_{n}, \omega_{u}}\left[\widetilde{H}_{x_{n}}>T_{U}\right]} \\
& \times \sum_{|e|=1}\left(\left(\omega^{\prime}\left(x_{n}, e\right)-\omega\left(x_{n}, e\right)\right)\right. \\
& \times\left[e_{1} \cdot e+E_{x_{n}+e, \omega_{u}}\left[\sum_{0}^{H_{x_{n}} \wedge T_{U}-1}\left(d \cdot e_{1}\right)\left(X_{k}, \omega_{u}\right)\right]\right. \\
& \left.\left.\quad-E_{x_{n}, \omega_{u}}\left[\sum_{0}^{\left[\widetilde{H}_{x_{n}} \wedge T_{U}-1\right.}\left(d \cdot e_{1}\right)\left(X_{k}, \omega_{u}\right)\right] \frac{P_{x_{n}+e, \omega_{u}}\left[H_{x_{n}}>T_{U}\right]}{P_{x_{n}, \omega_{u}}\left[\widetilde{H}_{x_{n}}>T_{U}\right]}\right]\right) .
\end{aligned}
$$

By the same calculation as in (3.8),

$$
\frac{P_{0, \omega_{u}}\left[H_{x_{n}}<T_{U}\right]}{P_{x_{n}, \omega_{u}}\left[\widetilde{H}_{x_{n}}>T_{U}\right]}=g_{U}\left(0, x_{n}, \omega_{u}\right) \stackrel{(2.30)}{\leq} c_{7} g_{0, U}\left(0, x_{n}\right)^{1 /(2-\alpha)} .
$$

Note that using the strong Markov property at time $H_{x_{n}} \wedge T_{U}$, we find for $|e|=1$,

$$
\begin{aligned}
& E_{x_{n}+e, \omega_{u}}\left[\sum_{0}^{H_{x_{n}} \wedge T_{U}-1}\left(d \cdot e_{1}\right)\left(X_{k}, \omega_{u}\right)\right] \\
& \quad=G_{U}\left(d \cdot e_{1}\right)\left(x_{n}+e, \omega_{u}\right)-P_{x_{n}+e, \omega_{u}}\left[H_{x_{n}}<T_{U}\right] G_{U}\left(d \cdot e_{1}\right)\left(x_{n}, \omega_{u}\right)
\end{aligned}
$$


and that

$$
G_{U}\left(d \cdot e_{1}\right)\left(x_{n}, \omega_{u}\right)=E_{x_{n}, \omega_{u}}\left[\sum_{0}^{\widetilde{H}_{x_{n}} \wedge T_{U}-1}\left(d \cdot e_{1}\right)\left(X_{k}, \omega_{u}\right)\right] P_{x_{n}, \omega_{u}}\left[\widetilde{H}_{x_{n}}>T_{U}\right]^{-1} \text {. }
$$

As a result the expression inside the square brackets in (3.43) equals

$$
\begin{aligned}
& e_{1} \cdot e+G_{U}\left(d \cdot e_{1}\right)\left(x_{n}+e, \omega_{u}\right) \\
& -G_{U}\left(d \cdot e_{1}\right)\left(x_{n}, \omega_{u}\right)\left(P_{x_{n}+e, \omega_{u}}\left[H_{x_{n}}<T_{U}\right]+P_{x_{n}+e, \omega_{u}}\left[H_{x_{n}}>T_{U}\right]\right) \\
& \quad=e_{1} \cdot e+\nabla_{e} G_{U}\left(d \cdot e_{1}\right)\left(x_{n}, \omega_{u}\right) .
\end{aligned}
$$

In view of (2.21) and (2.22), the absolute value of the above is smaller than $1+\frac{4}{3} c_{2} \frac{\varepsilon}{2 d} L \stackrel{(2.20)}{\leq} c_{14}(d)$. Since $\sum_{|e|=1}\left|\omega^{\prime}\left(x_{n}, e\right)-\omega\left(x_{n}, e\right)\right| \stackrel{(2.2)}{\leq} \varepsilon$, it follows from (3.43) and (3.44) and the above that for $n \geq 1, \omega, \omega^{\prime}$ in $\Omega_{\varepsilon}$ which coincide at each $x_{i}, i \neq n$,

$$
\left|\Gamma_{n}\left(\omega, \omega^{\prime}\right)\right| \leq c_{15}(d) \varepsilon g_{0, U}\left(0, x_{n}\right)^{1 /(2-\alpha)} .
$$

The claim (3.38) is then an easy consequence since $H_{n}$ and $H_{n-1}$ are the respective integrals of $G_{U}\left(d \cdot e_{1}\right)(0)$ with respect to the variables $\omega\left(x_{i}, \cdot\right), i>n$, and $\omega\left(x_{i}, \cdot\right)$, $i \geq n$, under the infinite product of the single site distribution $\mu$.

This completes the proof of (3.38) and hence of Proposition 3.2.

4. Denouement. The main purpose of this section is to apply the considerations of the previous sections to the proof of the main result of the present article [cf. (0.9)].

THEOREM $4.1(d \geq 3)$. For any $\eta \in(0,1)$, there exists $\varepsilon_{0}(d, \eta) \in(0,1)$ such that for $0<\varepsilon<\varepsilon_{0}$, and any $\mu$ supported on $\S_{\varepsilon}$, with

$$
\lambda \stackrel{\text { def }}{=} \mathbb{E}\left[d(0, \omega) \cdot e_{1}\right] \geq \lambda_{0}(d, \eta, \varepsilon) \stackrel{\text { def }}{=} \begin{cases}\varepsilon^{5 / 2-\eta}, & \text { when } d=3, \\ \varepsilon^{3-\eta}, & \text { when } d \geq 4,\end{cases}
$$

condition $\left(\mathrm{T}^{\prime}\right)$ holds relative to the direction $e_{1}$.

PROOF. We are going to check the effective criterion (0.12) with the help of the estimate (1.31) of Corollary 1.3, where we choose $a=\frac{1}{2}$, and we recall that in our context $\kappa=\frac{1}{4 d}$. We choose $\alpha \in[0,1)$ such that

$$
\frac{\eta}{8}=\frac{1-\alpha}{2-\alpha} \text {. }
$$

We also assume that $\varepsilon<\varepsilon_{1}(d, \eta)$, so that $\varepsilon 8 d<1$,

$$
\frac{L}{2} \stackrel{\text { def }}{=}\left[\frac{1}{2 \varepsilon} \min \left(\frac{3 d}{8 c_{1}}, \frac{1}{4 c_{2}}, \frac{\sqrt{c}_{6}}{4} \frac{1-\alpha}{2-\alpha}\right)\right] \geq 1
$$


and so that the assumptions of Propositions 3.1 and 3.2 are fulfilled and $L$ is an even integer as assumed throughout Section 1. Let us now specify the choices of parameters which appear in Theorem 1.1 and Corollary 1.3. We pick

$$
N=L^{3} \quad \text { [cf. (1.3)], }
$$

$$
h=\left[\frac{4}{c_{6}} L^{2} \log L\right], \quad H=\left[\frac{\log 2}{6400 \log 4 d}(N L)^{2}\right]
$$

By choosing $\varepsilon \leq \varepsilon_{2}(d, \eta) \leq \varepsilon_{1}(d, \eta)$, we can make sure that (1.9) and $\delta>2$ [cf. (1.14)].

Observe that since $\varepsilon L<\frac{3}{4}$ [cf. (4.3) and recall $c_{1}=d$ ],

$$
\left|G_{U}\left(d \cdot e_{1}\right)\right|_{\infty} \stackrel{(2.2),(2.21),(2.22)}{\leq} \frac{4}{3} \frac{\varepsilon}{2 d} c_{1} L^{2}<\frac{L}{2} .
$$

As a result [cf. (1.7) and (3.1)],

$$
\widehat{\rho}(0) \leq 3,
$$

and as a result we do not have to worry about any small denominator problem when bounding $\mathbb{E}[\widehat{\rho}(0)]$. Using (3.3), for $x \in \mathbb{Z}^{d}$ with $x \cdot e_{1}=0$,

$$
\mathbb{E}\left[G_{U}\left(d \cdot e_{1}\right)(x)\right] \geq \frac{2}{5} c_{1} \lambda L^{2} \stackrel{(4.1)}{\geq} \frac{2}{5} c_{1} \lambda_{0} L^{2},
$$

and hence using (4.8),

$$
\mathbb{E}[\widehat{\rho}(0)] \leq \frac{1-(1 / 5) c_{1} \lambda_{0} L}{1+(1 / 5) c_{1} \lambda_{0} L}
$$

$$
\begin{aligned}
& +3 \mathbb{P}\left[\inf _{\substack{x \cdot e_{1}=0 \\
\sup _{j \geq 2}\left|x \cdot e_{j}\right|<(N L)^{3} / 4}}\left\{G_{U}\left(d \cdot e_{1}\right)(x)-\mathbb{E}\left[G_{U}\left(d \cdot e_{1}\right)(x)\right]\right\}\right. \\
& \left.\leq-\frac{1}{5} c_{1} \lambda_{0} L^{2}\right] \\
& \stackrel{(3.33)}{\leq} 1-\frac{1}{5} c_{1} \lambda_{0} L+6\left\{\frac{(N L)^{3}}{2}\right\}^{d-1} \exp \left\{-\frac{1}{25} c_{1}^{2} \frac{\lambda_{0}^{2} L^{4}}{c_{\alpha, L}}\right\}
\end{aligned}
$$

and provided $\varepsilon<\varepsilon_{3}(d, \eta) \leq \varepsilon_{2}(d, \eta)$, in view of (4.1)-(4.3) and (3.35),

$$
\leq 1-\frac{1}{10} c_{1} \lambda_{0} L .
$$


We now turn to the quantity $p$ of (1.12) and (1.31). We will bound it from below. In the notation of (1.11), since $T_{U} \geq S, P_{0, \omega}$-a.s.,

$$
\begin{aligned}
\Delta(0, \omega) & =E_{0, \omega}\left[X_{S}, T_{U}=S\right]+E_{0, \omega}\left[X_{S}, T_{U}>S\right] \\
& =E_{0, \omega}\left[X_{T_{U}}, T_{U}=S\right]+E_{0, \omega}\left[X_{S}, T_{U}>S\right]
\end{aligned}
$$

so that

$$
\Delta(0, \omega)-E_{0, \omega}\left[X_{T_{U}}\right]=E_{0, \omega}\left[X_{S}-X_{T_{U}}, T_{U}>S\right]
$$

and of course the left-hand side of $(4.11)$ coincides with $\Delta(0, \omega)-G_{U}(d(\cdot, \omega))(0)$. We now see that

$$
\begin{aligned}
& \left|\Delta(0, \omega) \cdot e_{1}-G_{U}\left(d \cdot e_{1}\right)(0)\right| \\
& \quad \leq 2 L P_{0, \omega}\left[T_{U}>S\right] \leq 2 L P_{0, \omega}\left[T_{U}>h\right] \\
& \stackrel{(2.23)}{\leq} 4 L \exp \left\{-\frac{c_{6}}{L^{2}} h\right\} \stackrel{(4.5)}{\leq} 4 L^{-2} \leq \frac{1}{5} c_{1} \lambda_{0} L^{2},
\end{aligned}
$$

provided $\varepsilon \leq \varepsilon_{4}(d, \eta) \leq \varepsilon_{3}(d, \eta)$. As a result for $\varepsilon \leq \varepsilon_{5}(d, \eta) \leq \varepsilon_{4}(d, \eta)$,

$$
\begin{gathered}
p=\inf _{j \geq 2} \mathbb{P}\left[\forall z \in \widetilde{B}^{j}, \Delta(z, \omega) \cdot e_{1} \geq \gamma_{1},\left|\Delta(z, \omega) \cdot e_{i}\right| \leq \gamma_{2} h, \forall i \geq 2\right] \\
\text { (4.13) } \geq 1-\sup _{j \geq 2}\left|\widetilde{B}^{j}\right| \mathbb{P}\left[G_{U}\left(d \cdot e_{1}\right)-\mathbb{E}\left[G_{U}\left(d \cdot e_{1}\right)(0)\right] \leq-\frac{1}{10} c_{1} \lambda_{0} L^{2}\right] \\
\stackrel{(3.33)}{\geq} 1-(N L)^{2 d} 2 \exp \left\{-\frac{1}{100} \frac{c_{1}^{2} \lambda_{0}^{2} L^{4}}{c_{\alpha, L}}\right\} \geq 1-\exp \left\{-L^{\eta / 2}\right\} .
\end{gathered}
$$

Hence assuming $0<\varepsilon<\varepsilon_{5}$, it follows from (1.31) that

$$
\begin{aligned}
\mathbb{E}\left[\sqrt{\rho_{B_{0}}}\right] \leq(4 d)^{2}[ & \frac{2\left(1-\left(c_{1} / 10\right) \lambda_{0} L\right)^{N / 2}}{1-\sqrt{1-\left(c_{1} / 10\right) \lambda_{0} L}} \\
& \left.+2 d(4 d)^{(N L) / 2} \exp \left\{-\frac{M}{2}\left(p-\frac{7 N L}{M} \frac{\log 4 d}{\log 2}\right)_{+}^{2}\right\}\right] .
\end{aligned}
$$

Observe that for $0<\varepsilon<\varepsilon_{6}(d, \eta) \leq \varepsilon_{5}(d, \eta)$,

$$
M \geq \frac{100 \log 4 d}{\log 2} N L
$$

and

$$
\begin{aligned}
\mathbb{E}\left[\sqrt{\rho_{B_{0}}}\right] \leq(4 d)^{2}[ & 80\left(c_{1} \lambda_{0} L\right)^{-1} \exp \left\{-\frac{c_{1}}{20} N \lambda_{0} L\right\} \\
& \left.+2 d \exp \left\{N L\left[\frac{\log 4 d}{2}-\frac{100 \log 4 d}{2 \log 2}\left(\frac{3}{4}-\frac{7}{100}\right)^{2}\right]\right\}\right] .
\end{aligned}
$$


Recall that in the notation of (0.12), $L_{0}=N L=L^{4}, \widetilde{L}_{0}=\frac{1}{4}(N L)^{3}=\frac{1}{4} L^{12}$. We hence see that for $\varepsilon<\varepsilon_{0}(d, \eta)<\varepsilon_{6}(d, \eta)$, (0.12) is fulfilled and therefore condition $\left(\mathrm{T}^{\prime}\right)$ relative to $e_{1}$ holds true.

REMARK 4.2. We can of course replace (4.1) by

$$
|\mathbb{E}[d(0, \omega)]| \geq \begin{cases}\varepsilon^{5 / 2-\eta}, & \text { when } d \geq 3, \\ \varepsilon^{3-\eta}, & \text { when } d \geq 4,\end{cases}
$$

and choosing a possibly smaller $\widetilde{\varepsilon}_{0}(d, \eta) \leq \varepsilon_{0}(d, \eta)$ conclude that when $\varepsilon<\widetilde{\varepsilon}_{0}(d, \eta)$ then condition ( $\left.\mathrm{T}^{\prime}\right)$ holds with respect to some direction $e \in \mathbb{Z}^{d}$ with $|e|=1$.

5. About Kalikow's condition. In Sznitman and Zerner [2], an assumption previously introduced by Kalikow in [7] was nicknamed Kalikow's condition. This condition was later shown in [20] to imply condition (T), which itself trivially implies $\left(\mathrm{T}^{\prime}\right)$. We will provide here examples for which Kalikow's condition fails in a quite strong sense and yet $\left(\mathrm{T}^{\prime}\right)$ holds. Let us more precisely recall what Kalikow's condition is. Assume (0.1) and introduce for each $U$ strict subset of $\mathbb{Z}^{d}$, connected and containing 0 the auxiliary Markov chain on $U \cup \partial U$ with transition probability

$$
\begin{aligned}
\widehat{P}_{U}(x, x+e) & =\frac{\mathbb{E}\left[g_{U}(0, x, \omega) \omega(x, e)\right]}{\mathbb{E}\left[g_{U}(0, x, \omega)\right]}, \quad x \in U,|e|=1, \\
\widehat{P}_{U}(x, x) & =1 \quad \text { if } x \in \partial U,
\end{aligned}
$$

with $g_{U}$ defined by $(0.17)$ with $U$ as above [and not as in (0.16)]. Thanks to $(0.1)$ and the connectedness of $U \subsetneq \mathbb{Z}^{d},(5.1)$ is easily seen to make sense. Further, for $x \in U \cup \partial U$, one can consider the auxiliary local drift at $x \in U \cup \partial U$,

$$
\widehat{d}_{U}(x)= \begin{cases}\frac{\mathbb{E}\left[g_{U}(0, x, \omega) d(x, \omega)\right]}{\mathbb{E}\left[g_{U}(0, x, \omega)\right]}, & x \in U, \\ 0, & x \in \partial U .\end{cases}
$$

Kalikow's condition relative to $\ell \in S^{d-1}$ is the requirement

$$
\varepsilon(\ell, \mu) \stackrel{\text { def }}{=} \inf _{U, x \in U} \widehat{d}_{U}(x) \cdot \ell>0
$$

This condition is of course not very explicit, but Kalikow provided an explicit criterion in [7] which enables checking (5.3) and showed that (5.3) implied transience in the direction $\ell$, that is, $P_{0}\left[\lim X_{N} \cdot \ell=\infty\right]=1$. Further, consequences of (5.3) such as a ballistic law of large numbers, a central limit theorem and various large deviation controls were later proved in [22, 19]. As shown in Remark 2.5 of [22], Kalikow's condition characterizes walks with nondegenerate asymptotic velocity, when $d=1$. The results of this section will in particular imply that this is not the case when $d \geq 3$. 
To shed some light on the role of (5.3), it may be helpful to mention that the auxiliary Markov chains with transition probabilities given in (5.1) have the remarkable property that under $P_{0}$ and under the auxiliary chain starting at 0 , the walk has the same exit distribution from $U$, provided $U$ is almost surely left by the auxiliary chain. The interest of (5.3) is matter-of-factly to enable the control of exit distributions of the auxiliary chain and hence of the walk under $P_{0}$.

Let us now turn to the description of the class of walks under consideration in what follows. We assume that $d \geq 2$, and

$\mu$ is supported by $\delta_{\varepsilon}$ and invariant under the rotations when $d \geq 3$, respectively, the isometries when $d=2$, preserving $\mathbb{Z}^{d}$ and $e_{1}$.

Further, we have $\rho \in(0,1)$, such that

$$
\begin{aligned}
& \operatorname{var}_{\mathbb{P}}\left(\omega\left(0, e_{1}\right)\right)=\operatorname{var}_{\mathbb{P}}\left(\omega\left(0,-e_{1}\right)\right) \geq \rho \varepsilon^{2}, \\
& \operatorname{cov}_{\mathbb{P}}\left(\omega\left(0, e_{1}\right), \omega\left(0,-e_{1}\right)\right) \leq(1-\rho) \operatorname{var}_{\mathbb{P}}\left(\omega\left(0, e_{1}\right)\right) .
\end{aligned}
$$

An example corresponds to the situation mentioned in (0.10) of an isotropic law $\tilde{\mu}$ $(d \geq 3)$ supported on $\S_{\varepsilon / 2}$ for which

$$
\operatorname{var}_{\tilde{\mu}}\left(p\left(e_{1}\right)\right) \geq \rho \varepsilon^{2}, \quad \operatorname{cov}_{\tilde{\mu}}\left(p\left(e_{1}\right), p\left(-e_{1}\right)\right) \leq(1-\rho) \operatorname{var}_{\tilde{\mu}}\left(p\left(e_{1}\right)\right),
$$

and a law $\mu$ which is the image of $\tilde{\mu}$ under the map

$$
p(e) \rightarrow p(e)+\frac{\lambda}{2} e \cdot e_{1} \quad \text { for }|e|=1,
$$

with $|\lambda|<\frac{\varepsilon}{4 d}$. It is then straightforward to see that (5.4) and (5.5) are satisfied.

We will use the notation

$$
U_{+}=\left\{y \in \mathbb{Z}^{d}, y \cdot e_{1} \geq 0\right\}, \quad U_{-}=\left\{y \in \mathbb{Z}^{d}, y \cdot e_{1} \leq 0\right\} .
$$

In what follows we recall that $\kappa=\frac{1}{4 d}$.

THEOREM $5.1(d \geq 2)$. Let $\rho \in(0,1)$ and assume (5.4) and (5.5). Then for

$$
\varepsilon<\frac{1}{4} \kappa^{4} \rho^{2}, \quad|\lambda| \leq \frac{\kappa^{2}}{2} \rho^{2} \varepsilon^{2} \quad \text { with } \lambda=\mathbb{E}\left[d(0, \omega) \cdot e_{1}\right],
$$

$$
\widehat{d}_{U_{+}}(0)=v_{+} e_{1}, \quad \widehat{d}_{U-}(0)=v_{-} e_{1} \quad \text { with } v_{+}>0, v_{-}<0 .
$$

In particular Kalikow's condition fails for every $\ell \in S^{d-1}$.

PROOF. Let $V$ stand for either $U_{+}$or $U_{-}$. From (5.4), using a rotation when $d \geq 3$, a symmetry when $d=2$, preserving $e_{1}$ and mapping $e_{i}$ into $-e_{i}$, for a given $i \geq 2$, we see that

$$
\widehat{d}_{V}(0) \cdot e_{i}=0 \quad \text { for } i \geq 2
$$


and hence $\widehat{d}_{V}(0)$ is colinear to $e_{1}$. We see by analogous manipulations as in (3.11)(3.14), that since $\frac{\varepsilon}{\kappa} \leq \frac{1}{2}$,

$$
\mathbb{E}\left[g_{V}(0,0, \omega)\left(d \cdot e_{1}\right)(0)\right]=\mathbb{E}\left[\frac{\left(d \cdot e_{1}\right)(0)}{P_{0, \omega}\left[\widetilde{H}_{0}>T_{V}\right]}\right]=\alpha_{V}+\beta_{V}+\gamma_{V},
$$

where in the notation of (3.10),

$$
\begin{aligned}
\alpha_{V} & =\lambda \mathbb{E}\left[P_{0, \omega}\left[\tilde{H}_{0}>T_{V}\right]^{-1}\right] \\
\beta_{V} & =\mathbb{E}\left[\frac{\left(\tilde{\delta}\left(0, e_{1}\right)-\tilde{\delta}\left(0,-e_{1}\right)\right)}{\bar{P}_{0, \omega}\left[\widetilde{H}_{0}>T_{V}\right]^{2}} \sum_{|e|=1} \widetilde{\delta}(0, e) P_{e, \omega}\left[H_{0}<T_{V}\right]\right] \\
\left|\gamma_{V}\right| & \leq 2\left(\frac{\varepsilon}{\kappa}\right)^{3} .
\end{aligned}
$$

Using independence,

$$
\beta_{V}=\sum_{|e|=1} \mathbb{E}\left[\left(\tilde{\delta}\left(0, e_{1}\right)-\tilde{\delta}\left(0,-e_{-1}\right)\right) \tilde{\delta}(0, e)\right] \mathbb{E}\left[\frac{P_{e, \omega}\left[H_{0}<T_{V}\right]}{\bar{P}_{0, \omega}\left[\widetilde{H}_{0}>T_{V}\right]^{2}}\right] .
$$

In view of (5.4) the rightmost term in the above expression is the same for all $|e|=1$ with $e \cdot e_{1}=0$. Since $\sum_{e \cdot e_{1}=0} \tilde{\delta}(0, e)=-\left(\tilde{\delta}\left(0, e_{1}\right)+\widetilde{\delta}\left(0,-e_{1}\right)\right)$, we find

$$
\begin{aligned}
\beta_{V}= & -\mathbb{E}\left[\left(\widetilde{\delta}\left(0, e_{1}\right)-\tilde{\delta}\left(0,-e_{-1}\right)\right)\left(\widetilde{\delta}\left(0, e_{1}\right)+\widetilde{\delta}\left(0,-e_{-1}\right)\right)\right] \mathbb{E}\left[\frac{P_{e_{2}, \omega}\left[H_{0}<T_{V}\right]}{\bar{P}_{0, \omega}\left[\widetilde{H}_{0}>T_{V}\right]^{2}}\right] \\
& +\sum_{e \cdot e_{1}= \pm 1} \mathbb{E}\left[\left(\widetilde{\delta}\left(0, e_{1}\right)-\tilde{\delta}\left(0,-e_{1}\right)\right) \widetilde{\delta}(0, e)\right] \mathbb{E}\left[\frac{P_{e, \omega}\left[H_{0}<T_{V}\right]}{\bar{P}_{0, \omega}\left[\widetilde{H}_{0}>T_{V}\right]^{2}}\right] .
\end{aligned}
$$

In view of the first equality of (5.5), the first expectation in the right-hand side vanishes and

$$
\begin{aligned}
\beta_{V}= & {\left[\operatorname{var}_{\mathbb{P}}\left(\omega\left(0, e_{1}\right)\right)-\operatorname{cov}_{\mathbb{P}}\left(\omega\left(0, e_{1}\right), \omega\left(0,-e_{1}\right)\right)\right] } \\
& \times \mathbb{E}\left[\frac{P_{e_{1}, \omega}\left[H_{0}<T_{V}\right]-P_{-e_{1}, \omega}\left[H_{0}<T_{V}\right]}{\bar{P}_{0, \omega}\left[\widetilde{H}_{0}>T_{V}\right]^{2}}\right] .
\end{aligned}
$$

As a result of (5.5), the first factor is bigger than $\rho^{2} \varepsilon^{2}$. We hence see that

$$
\beta_{U_{+}} \geq \kappa \rho^{2} \varepsilon^{2}, \quad \beta_{U_{-}} \leq-\kappa \rho^{2} \varepsilon^{2} .
$$

Moreover, from (5.8),

$$
\left|\alpha_{V}\right| \leq \frac{|\lambda|}{\kappa} \leq \frac{\kappa}{2} \rho^{2} \varepsilon^{2}
$$

We thus see that

$$
\mathbb{E}\left[g_{U_{+}}(0,0, \omega)\left(d \cdot e_{1}\right)(0)\right] \geq \frac{\kappa}{2} \rho^{2} \varepsilon^{2}-2\left(\frac{\varepsilon}{\kappa}\right)^{3}=\varepsilon^{2}\left(\frac{\kappa}{2} \rho^{2}-\frac{2 \varepsilon}{\kappa^{3}}\right)>0,
$$


whereas

$$
\mathbb{E}\left[g_{U_{-}}(0,0, \omega)\left(d \cdot e_{1}\right)(0)\right] \leq-\frac{\kappa}{2} \rho^{2} \varepsilon^{2}+2\left(\frac{\varepsilon}{\kappa}\right)^{3}<0 .
$$

This completes the proof of (5.9).

REMARK 5.2. (1) One can of course relax some of the assumptions of Theorem 5.1, which by no means is the most general result one can obtain. Let us however note that one cannot simply drop the last inequality of (5.5). Indeed if one constructs $\mu$ as indicated in (5.6) with $\tilde{\mu}$ isotropic concentrated on $\delta_{\varepsilon / 2} \cap\left\{(p(e))_{|e|=1} ; p(e)=p(-e)\right.$, for $\left.|e|=1\right\}$, such that $\operatorname{var}_{\tilde{\mu}}(p(e)) \geq \rho \varepsilon^{2}$, then the local drift at any site in a typical $\mu^{\otimes \mathbb{Z}^{d}}$-configuration is deterministic and equal to $\lambda e_{1}$. Hence Kalikow's condition (5.3) holds relative to $\frac{\lambda}{|\lambda|} e_{1}$, provided $\lambda \neq 0$.

(2) In Remark 2.5 of [20] one can find another example of walk for which Kalikow's condition fails. However the proof used in [20] relies on the calculation of $\widehat{d}_{U}(0)$, for $U=\{0\}$ and $U=\left\{-e_{1}, 0\right\}$, whereas (5.9) offers a more fundamental obstruction to Kalikow's condition and the way it is used in proofs. As we now will see the class described in Theorem 5.1 contains examples of walks with ballistic behavior when $d \geq 3$.

Corollary $5.3(d \geq 3)$. Assume $\rho, \eta \in(0,1)$, there exists $\widetilde{\varepsilon}(d, \eta, \rho) \in$ $(0,1)$ such that when $0<\varepsilon<\tilde{\varepsilon}$, (5.4) and (5.5) hold and

$$
\begin{array}{ll}
\varepsilon^{5 / 2-\eta}<\lambda<\frac{\kappa^{2}}{2} \rho^{2} \varepsilon^{2} & \text { when } d=3, \\
\varepsilon^{3-\eta}<\lambda<\frac{\kappa^{2}}{2} \rho^{2} \varepsilon^{2} & \text { when } d \geq 4,
\end{array}
$$

condition $\left(\mathrm{T}^{\prime}\right)$ holds relative to $e_{1}$ together with (5.9), and Kalikow's condition fails with respect to all directions.

The above corollary shows that $\left(\mathrm{T}^{\prime}\right)$ may very well hold in spite of an "oscillatory" behavior of the auxiliary drift.

\section{APPENDIX}

This appendix is devoted to the proof of Lemma 1.2, which is a variation on the derivation of (2.3.9) in [21], and to the proof of the Green function estimates (2.11) and (2.12). We begin with the following.

ProOf OF Lemma 1.2. We use the notation of Section 1. For $a \leq b$ in $\mathbb{Z}$ we introduce

$$
\pi_{a, b}=\prod_{a<i \leq b} \widehat{\rho}(i, \omega) .
$$


We will first prove that in the notations of (1.16), below (1.6) and (1.3),

(A.2) $\quad P_{0, \omega}\left[\widetilde{T}_{-N L}<\widetilde{T} \wedge T_{N L}\right] \leq \frac{\pi_{-N, 0}+\cdots+\pi_{-N, N-1}}{1+\pi_{-N,-N+1}+\cdots+\pi_{-N, N-1}}$.

To this end we define $f(i, \omega)$ for $i \leq N, \omega \in \Omega$, via

$$
\begin{aligned}
& f(N, \omega)=0, \\
& f(i, \omega)=\sum_{i \leq m<N} \prod_{m<j<N} \widehat{\rho}(j, \omega)^{-1} \quad \text { for } i<N .
\end{aligned}
$$

We denote by $V_{k}, k \geq 0$, the sequence of iterates of the stopping time $V=$ $T_{X_{0} \cdot e_{1}+L} \wedge \widetilde{T}_{X_{0} \cdot e_{1}-L}$, namely,

$$
V_{0}=0 \quad \text { and } \quad \text { for } k \geq 0, \quad V_{k+1}=V \circ \theta_{V_{k}}+V_{k} .
$$

We write

$$
\tau=\inf \left\{k \geq 0,\left|X_{V_{k}} \cdot e_{1}\right| \geq N L\right\}
$$

Note that with definition (1.8),

(A.6) $\quad P_{0, \omega}$-a.s. on $\left\{\widetilde{T}_{-N L}<\widetilde{T} \wedge T_{N L}\right\}, \quad X_{V_{\tau}} \in \mathscr{H}_{-N} \quad$ and $\quad V_{\tau}<\widetilde{T}$.

Moreover for $m \geq 0$,

$$
\begin{aligned}
E_{0, \omega}[ & \left.f\left(\frac{1}{L} X_{V_{(m+1) \wedge \tau}} \cdot e_{1}\right), V_{(m+1) \wedge \tau} \leq \widetilde{T}\right] \\
\leq & E_{0, \omega}\left[f\left(\frac{1}{L} X_{V_{m \wedge \tau}} \cdot e_{1}\right), V_{m \wedge \tau} \leq \widetilde{T}, \tau \leq m\right] \\
& +E_{0, \omega}\left[f\left(\frac{1}{L} X_{V_{m+1}} \cdot e_{1}\right), V_{m}<\widetilde{T}, \tau>m\right]
\end{aligned}
$$

so that using the strong Markov property

$$
\begin{aligned}
= & E_{0, \omega}\left[f\left(\frac{1}{L} X_{V_{m}} \cdot e_{1}\right), V_{m \wedge \tau} \leq \widetilde{T}, \tau \leq m\right] \\
& +E_{0, \omega}\left[\tau>m, V_{m}<\widetilde{T}, E_{X_{V_{m}}, \omega}\left[f\left(\frac{1}{L} X_{V_{1}} \cdot e_{1}\right)\right]\right] .
\end{aligned}
$$


However on $\left\{\tau>m, V_{m}<T\right\}, P_{0, \omega}$-a.s.,

$$
\begin{aligned}
& E_{X_{V_{m}}, \omega}\left[f\left(\frac{1}{L} X_{V_{1}} \cdot e_{1}\right)\right] \\
& =f\left(\frac{1}{L} X_{V_{m}} \cdot e_{1}\right)+\widehat{p}\left(X_{V_{m}}, \omega\right)\left[f\left(\frac{1}{L} X_{V_{m}} \cdot e_{1}+1\right)-f\left(\frac{1}{L} X_{V_{m}} \cdot e_{1}\right)\right] \\
& +\widehat{q}\left(X_{V_{m}}, \omega\right)\left[f\left(\frac{1}{L} X_{V_{m}} \cdot e_{1}-1\right)-f\left(\frac{1}{L} X_{V_{m}} \cdot e_{1}\right)\right] \\
& \stackrel{(\text { A.3) }}{=} f\left(\frac{1}{L} X_{V_{m}} \cdot e_{1}\right) \\
& +\prod_{\frac{1}{L} X_{V_{m}} \cdot e_{1}<j<N} \widehat{\rho}(j, \omega)^{-1}\left[-\widehat{p}\left(X_{V_{m}}, \omega\right)+\widehat{q}\left(X_{V_{m}}, \omega\right) \widehat{\rho}\left(\frac{1}{L} X_{V_{m}} \cdot e_{1}\right)^{-1}\right] \\
& \stackrel{(1.7)}{\leq} f\left(\frac{1}{L} X_{V_{m}} \cdot e_{1}\right) \text {. }
\end{aligned}
$$

(A.8)

Coming back to the last line of (A.7), we see that

$$
\begin{aligned}
& E_{0, \omega}\left[f\left(\frac{1}{L} X_{V_{(m+1) \wedge \tau}}\right), V_{(m+1) \wedge \tau} \leq \widetilde{T}\right] \\
& \quad \leq E_{0, \omega}\left[f\left(\frac{1}{L} X_{V_{m}} \cdot e_{1}\right), V_{m \wedge \tau} \leq \widetilde{T}\right] \leq f(0)
\end{aligned}
$$

by induction. Since $\tau$ is $P_{0, \omega}$-a.s. finite, it then follows from Fatou's lemma that

$$
E_{0, \omega}\left[f\left(\frac{1}{L} X_{V_{\tau}} \cdot e_{1}\right), V_{\tau} \leq \tilde{T}\right] \leq f(0) .
$$

From this we deduce that

$$
\begin{aligned}
P_{0, \omega}\left[\tilde{T}_{-N L}<\tilde{T} \wedge T_{N L}\right] & \leq \frac{f(0)}{f(-N)} \\
& =\frac{\prod_{0<j<N} \widehat{\rho}(j, \omega)^{-1}+\prod_{1<j<N} \widehat{\rho}(j, \omega)^{-1}+\cdots+1}{\prod_{-N<j<N} \widehat{\rho}(j, \omega)^{-1}+\cdots+1} \\
& =\frac{\pi_{-N, 0}+\cdots+\pi_{-N, N-1}}{1+\pi_{-N,-N+1}+\cdots+\pi_{-N, N-1}},
\end{aligned}
$$

proving (A.2). We now define

$$
\text { (A.11) } \quad A=P_{0, \omega}\left[\tilde{T}_{-N L}<\tilde{T} \wedge T_{N L}\right]+P_{0, \omega}\left[\tilde{T} \leq T_{-N L} \wedge T_{N L}\right] \text {, }
$$

so that in the notation of (1.4) and (1.5),

$$
q_{B} \leq A \quad \text { and } \quad \rho_{B}=\frac{q_{B}}{1-q_{B}} \leq \frac{A}{(1-A)_{+}}
$$


using in the last step the fact that $q \rightarrow \frac{q}{1-q}$ is nondecreasing for $q<1$. As a result on $g$ [cf. (1.17)],

$$
A \leq \frac{\pi_{-N, 0}+\cdots+\pi_{-N, N-1}+\kappa^{6 N L}\left(1+\cdots+\pi_{-N, N-1}\right)}{1+\cdots+\pi_{-N, N-1}},
$$

$$
(1-A)_{+} \geq \frac{1+\cdots+\pi_{-N,-1}-\kappa^{6 N L}\left(1+\cdots+\pi_{-N, N-1}\right)}{1+\cdots+\pi_{-N, N-1}} .
$$

Moreover, as a result of $(0.1)$,

$$
\begin{aligned}
\kappa^{L} & \leq \widehat{\rho}(i, \omega) \leq \kappa^{-L} \quad \text { and } \\
\kappa^{2 N L} & \leq \pi_{-N, j} \leq \kappa^{-2 N L} \quad \text { for }-N<j<N .
\end{aligned}
$$

Further by assumption $L \geq 2 \geq \sup _{u \geq 0} 2 u\left(\frac{1}{2}\right)^{u}$, and since $\kappa \leq \frac{1}{2}$,

$$
6 N L \kappa^{3 N L} \leq L .
$$

As a result we see that

$$
(1-A)_{+} \geq \frac{1+\kappa^{L}-2 N \kappa^{4 N L}}{1+\cdots+\pi_{-N, N-1}} \geq\left(1+\cdots+\pi_{-N, N-1}\right)^{-1},
$$

and therefore

$$
\begin{aligned}
\rho_{B} & \leq \pi_{-N, 0}+\cdots+\pi_{-N, N-1}+\kappa^{6 N L}\left(1+\cdots+\pi_{-N, N-1}\right) \\
& \leq \pi_{-N, 0}+\cdots+\pi_{-N, N-1}+2 N \kappa^{4 N L} \leq 2\left(\pi_{-N, 0}+\cdots+\pi_{-N, N-1}\right),
\end{aligned}
$$

since $\pi_{-N, 0} \geq \kappa^{N L}$ and using (A.15).

Note that the variables $\widehat{\rho}(i, \omega)$, for $i$ even and for $i$ odd are two collections of i.i.d. variables. Since $a \in(0,1]$, we have

$$
\begin{aligned}
\mathbb{E}\left[\rho_{B}^{a}, \mathcal{g}\right] & \leq 2 \sum_{0 \leq m<N} \mathbb{E}\left[\pi_{-N, m}^{a}, \mathcal{g}\right] \\
& \leq 2 \sum_{0 \leq m<N} \prod_{-N<j \leq m} \mathbb{E}\left[\widehat{\rho}(j)^{2 a}\right]^{1 / 2} \\
& =2 \sum_{0 \leq m<N} \mathbb{E}\left[\widehat{\rho}(0)^{2 a}\right]^{(m+N) / 2} \leq \frac{2 \mathbb{E}\left[\widehat{\rho}(0)^{2 a}\right]^{N / 2}}{\left(1-\mathbb{E}\left[\widehat{\rho}(0)^{2 a}\right]^{1 / 2}\right)_{+}} .
\end{aligned}
$$

On the other hand, we know from (0.1) that

$$
\rho_{B} \leq \kappa^{-N L},
$$

and hence combining (A.17) and (A.18),

$$
\mathbb{E}\left[\rho_{B}^{a}\right] \leq \frac{2 \mathbb{E}\left[\widehat{\rho}(0)^{2 a}\right]^{N / 2}}{\left(1-\mathbb{E}\left[\widehat{\rho}(0)^{2 a}\right]^{1 / 2}\right)_{+}}+\kappa^{-a N L} \mathbb{P}\left[g^{c}\right],
$$

which completes the proof of Lemma 1.2. 
We now turn to Green's function estimates and will provide the following.

PROOF OF (2.11) AND (2.12). We first derive some pointwise bounds. It follows from (2.6) that for $x \in U, y \in U,|e|=1$,

$$
g_{0, U}(x+e, y)-g_{0, U}(x, y)
$$

$$
=\sum_{k \in \mathbb{Z}} \nabla_{e} g^{0}\left(x-y+2 k 2 L e_{1}\right)-\nabla_{e} g^{0}\left(x-y_{*}+(2 k+1) 2 L e_{1}\right),
$$

using the notation (2.19). From Lawler ([11], page 32, Theorem 1.5.5) we know that

$$
\left|\nabla_{e} g^{0}(z)\right| \leq \frac{c(d)}{(1+|z|)^{d-1}} \quad \text { for } z \in \mathbb{Z}^{d} .
$$

Hence for $x, y \in U$,

$$
\begin{aligned}
& \left|g_{0, U}(x+e, y)-g_{0, U}(x, y)\right| \\
& \leq c(d)\left[(1+|x-y|)^{-(d-1)}+\left(1+\left|x-y_{*}+2 L e_{1}\right|\right)^{-(d-1)}\right. \\
& \left.\quad+\left(1+\left|x-y_{*}-2 L e_{1}\right|\right)^{-(d-1)}+\sum_{|k| \geq 2}(k L)^{-(d-1)}\right] \\
& \leq c(d)\left[(1+|x-y|)^{-(d-1)}+\left(1+\left|x-y_{*}+2 L e_{1}\right|\right)^{-(d-1)}\right. \\
& \left.\quad+\left(1+\left|x-y_{*}-2 L e_{1}\right|\right)^{-(d-1)}+L^{-(d-1)}\right] .
\end{aligned}
$$

By an analogous argument as in the proof of (2.16), we find that for $x, y \in U$, $|e|=1$ with $\left|(x-y)_{\perp}\right|>L$, and $T$ as above (2.16),

$$
\begin{aligned}
& \left|g_{0, U}(x+e, y)-g_{0, U}(x, y)\right| \\
& =\left|E^{Q_{y}}\left[g_{0, U}\left(x+e, X_{T}\right)-g_{0, U}\left(x, X_{T}\right), T<T_{U}\right]\right| \\
& \leq \sup \left\{\left|g_{0, U}(x+e, z)-g_{0, U}(x, z)\right| ;\right. \\
& \left.\quad\left|(x-z)_{\perp}\right| \geq \frac{1}{2}\left|(x-y)_{\perp}\right|-1\right\} Q_{y}\left[T<T_{U}\right] .
\end{aligned}
$$

Combining the above estimate and (A.22) we find that for $x, y \in U,|e|=1$,

$$
\begin{aligned}
& \left|g_{0, U}(x+e, y)-g_{0, U}(x, y)\right| \\
& \leq c(d)\left[(1+|x-y|)^{-(d-1)}+\left(1+\mid x-y_{*}+2 L e_{1}\right)^{-(d-1)}\right. \\
& \left.\quad+\left(1+\left|x-y_{*}-2 L e_{1}\right|\right)^{-(d-1)}+L^{-(d-1)}\right] \\
& \quad \times \exp \left\{-\frac{c^{\prime}(d)}{L}\left|(x-y)_{\perp}\right|\right\} .
\end{aligned}
$$


Moreover, by symmetry of $g_{0, U}(\cdot, \cdot)$, a similar estimate holds as well for $\left|g_{0, U}(x, y+e)-g_{0, U}(x, y)\right|$ in place of $\left|g_{0, U}(x+e, y)-g_{0, U}(x, y)\right|$.

To obtain (2.11), we distinguish between the case $\left|(x-y)_{\perp}\right|>L$ and the case $\left|(x-y)_{\perp}\right| \leq L$, and see that for $x \in U,|e|=1$,

(A.24)

$$
\sum_{y \in U}\left(\left|g_{0, U}(x+e, y)-g_{0, U}(x, y)\right|+\left|g_{0, U}(x, y+e)-g_{0, U}(x, y)\right|\right)
$$

$$
\leq c(d) \sum_{y \in U,\left|(x-y)_{\perp}\right| \leq L}\left[(1+|x-y|)^{-(d-1)}\right.
$$

$$
+\left(1+\left|x-y_{*}+2 L e_{1}\right|\right)^{-(d-1)}
$$

$$
\begin{array}{r}
\left.+\left(1+\left|x-y_{*}-2 L e_{1}\right|\right)^{-(d-1)}+L^{-(d-1)}\right] \\
+c(d) \sum_{y \in U,|(x-y) \perp| \geq L} L^{-(d-1)} \exp \left\{-\frac{c^{\prime}(d)}{L}\left|(x-y)_{\perp}\right|\right\} \leq c(d) L,
\end{array}
$$

which proves (2.11), and in a similar fashion,

$$
\begin{aligned}
& \sum_{y \in U}\left[\left(g_{0, U}(x+e, y)-g_{0, U}(x, y)\right)^{2}+\left(g_{0, U}(x, y+e)-g_{0, U}(x, y)\right)^{2}\right] \\
& \leq c(d),
\end{aligned}
$$

so that (2.12) holds as well.

\section{REFERENCES}

[1] Alon, N., Spencer, J. and Erdös, P. (1992). The Probabilistic Method. Wiley, New York.

[2] Anshelevich, V. V., Khanin, K. M. and Sinai, Ya. G. (1982). Symmetric random walks in random environments. Comm. Math. Phys. 85 449-470.

[3] Bolthausen, E. and Sznitman, A. S. (2002). Ten Lectures on Random Media. Birkhäuser, Basel.

[4] Bolthausen, E., Sznitman, A. S. and Zeitouni, O. (2002). Cut points and diffusive random walks in random environment. Ann. Inst. H. Poincaré Probab. Statist. To appear.

[5] BRicmont, J. and Kupiainen, A. (1991). Random walks in asymmetric random environments. Comm. Math. Phys. 142 345-420.

[6] Hughes, B. D. (1996). Random Walks and Random Environments 2. Clarendon Press, Oxford.

[7] Kalikow, S. A. (1981). Generalized random walk in a random environment. Ann. Probab. 9 $753-768$.

[8] Khas'minskiI, R. Z. (1959). On positive solutions of the equation $A u+V u=0$. Theoret. Probab. Appl. 4 309-318.

[9] KozLov, S. M. (1985). The method of averaging and walks in inhomogeneous environments. Russian Math. Surveys 40 73-145.

[10] LAWLER, G. F. (1982). Weak convergence of a random walk in a random environment. Comm. Math. Phys. 87 81-87.

[11] LAWLER, G. F. (1991). Intersection of Random Walks. Birkhäuser, Basel.

[12] Molchanov, S. A. (1992). Lectures on Random Media. Ecole d'eté de Probabilités de St. Flour XXII. Lecture Notes in Math. 1581. Springer, Berlin. 
[13] Olla, O. (1994). Homogenization of Diffusion Processes in Random Fields. Ecole Polytechnique, Palaiseau.

[14] Papanicolaou, G. and Varadhan, S. R. S. (1981). Boundary value problems with rapidly oscillating random coefficients. In Random Fields (J. Fritz and D. Szasz, eds.) 835-873. North-Holland, Amsterdam.

[15] Papanicolaou, G. and Varadhan, S. R. S. (1982). Diffusion with Random Coefficients. Statistics and Probability: Essays in Honor of C.R. Rao (G. Kallianpur, P. R. Krishnajah and J. K. Gosh, eds.) 547-552. North-Holland, Amsterdam.

[16] Reed, M. and Simon, B. (1975). Methods of Modern Mathematical Physics 2. Academic Press, New York.

[17] Solomon, F. (1975). Random walk in random environment. Ann. Probab. 3 1-31.

[18] Sznitman, A. S. (1999). Slowdown and neutral pockets for a random walk in random environment. Probab. Theory Related Fields 115 287-323.

[19] Sznitman, A. S. (2000). Slowdown estimates and central limit theorem for random walks in random environment. J. Eur. Math. Soc. 2 93-143.

[20] Sznitman, A. S. (2001). On a class of transient random walks in random environment. Ann. Probab. 29 723-764.

[21] Sznitman, A. S. (2002). An effective criterion for ballistic behavior of random walks in random environment. Probab. Theory Related Fields 122 509-544.

[22] Sznitman, A. S. and Zerner, M. P. W. (1999). A law of large numbers for random walks in random environment. Ann. Probab. 27 1851-1869.

[23] Zeitouni, O. (2001). Notes of Saint Flour lectures 2001. Preprint. Available at wwwee.technion.ac.il/ $\sim$ zeitouni/ps/notes1.ps.

Departement MATHEMATiK

ETH-ZENTRUM

CH-8092 ZÜRICH

SWITZERLAND

E-MAIL: sznitman@math.ethz.ch 